\title{
Gain Scheduling Output Feedback Control of Linear Plants with Actuator Saturation
}

\author{
Xiaojun Ban* \\ Center for Control Theory and Guidance Technology \\ Harbin Institute of Technology, Harbin 150001, P. R. China \\ E-mail: banxiaojun@hit.edu.cn \\ Fen $\mathrm{Wu}$ \\ Department of Mechanical and Aerospace Engineering \\ North Carolina State University, Raleigh, NC 27695, USA \\ E-mail: fwu@ncsu.edu
}

\begin{abstract}
From a gain-scheduling perspective, we will study the output feedback control problem for linear systems with some of control channels subject to actuator saturation. This includes the scenario of all actuator saturation as a special case. A feedback controller, expressed in the form of linear fractional transformation, is proposed to guarantee regional stability of the closed-loop system and provide disturbance/error attenuation measured in $\mathcal{L}_{2}$ gain. The resulting synthesis condition is formulated as linear matrix inequalities (LMIs) and can be solved efficiently. Moreover, explicit formulas are derived to calculate controller gains, which reduces the computational cost compared to the method of directly solving the LMI-based condition. Numerical examples are provided to demonstrate the proposed saturation control approach.
\end{abstract}

Keywords Actuator saturation; gain-scheduling; output feedback; regional stability; disturbance attenuation; $\mathcal{L}_{2}$ gain.

\footnotetext{
*Corresponding author. Phone: (0451) 86402224-8206, Fax: (0451) 86402689.

${ }^{0}$ Xiaojun Ban's work is supported by the National Natural Science Foundation of China (NSFC) under Grant No. 61304006 and 61273095. Fen Wu's work is supported in part by the NSF Grant CMMI-0800044.
} 


\section{Introduction}

Saturation nonlinearity is widely encountered in control engineering and can significantly deteriorate the performance of a closed-loop system or even render a stable system unstable. Therefore it has attracted a lot of attention from the control community (see, for example, [2, 9, 14, 23] and the references therein). This literature covers a wide range of topics, including stabilization, output regulation, and disturbance rejection. Earlier research focuses on stable open-loop systems, for which various control problems have been studied in depth in the global or semi-global framework (see, for example, [14, 3, 15, 16, 20, 22]).

More recent literature has witnessed a shift of focus to exponentially unstable open-loop systems. Indeed many control systems with actuator saturation, such as flight control, are exponentially unstable. As such systems under actuator saturation are null controllable only in a part of the state space, the objectives here are to characterize the null controllable region [9] and to design feedback laws that work on the entire null controllable region or a large portion of it (see, for example, $[8,17,9,19,10,5])$. Different control design procedures based on rigorous theoretical analysis have been proposed through various framework. Nevertheless, most of this literature on exponentially unstable systems pertains to state feedback. A few exceptions where output feedback are used include $[10,17,19,21,25,26,4]$. In particular, a saturation control synthesis method was proposed in [4] for the construction of output feedback controllers with an internal deadzone loop. It has been shown the synthesis condition for such a controller can be formulated into the LMI form. In [26], a gain-scheduled saturation controller in the output feedback form has been developed to attenuate the effect of the disturbances on the system output in addition to achieving local stability. Nevertheless, the control synthesis conditions are in bilinear matrix inequality forms and not easy to solve. On the basis of the work in [26], by carefully choosing the auxiliary subspace in representing the saturation/deadzone nonlinearities, the output feedback synthesis condition can be recast into linear matrix inequalities [27]. However, the disadvantage is that the form of the controller is somewhat complicated and the synthesis condition is also computationally expensive. It consists of $2^{n_{u}}+n_{u}+1$ LMIs and $2+n_{u}+2 \times 2^{n_{u}}$ decision variables with $n_{u}$ representing the number of inputs.

This research is inspired by the systematic gain-scheduling control design techniques developed in $[18,1]$. When the parameter dependency in both plant and controller is in the form of LFT (linear fractional transformation), it was demonstrated that the gain-scheduling control problem can be reformulated as one of robust performance problems with a special plant/uncertainty structure. As a result, the underlying control synthesis is a convex problem and the existence of such a gainscheduling controller is fully characterized by a set of LMIs. This promising control structure is applicable whenever the values of parameters are measurable in real-time.

According to the above idea, the linear system with input saturation will be recast into the form of LFT through deadzone functions and a gain-scheduling controller with the same parameter dependency will be introduced. Consequently, a gain-scheduling output feedback controller in the form of LFT will be designed for saturated control systems to guarantee the stability of the closed-loop system and provide the disturbance/error attenuation as measured in $\mathcal{L}_{2}$ gain. The resulting controller is time-varying and smoothly scheduled by the measured parameters. The synthesis condition is formulated as a convex optimization problem in terms of LMIs for which efficient optimization techniques are available. Explicit formulae are also derived to calculate the controller gains, which reduces the computational cost compared to the method of directly solving the LMI-based condition. The proposed gain-scheduling controller has much simpler structure 
than the one used in [27]. Moreover, it can be shown the saturation controller presented in [4] is just a special form of the controller proposed in this research. In addition, in this research, we will focus on the linear plants with partial control channels subject to actuator saturation, which includes the plants with all the input channels saturated as a special case. All together, our proposed approach provides an alternative approach to the existing actuator saturation control methods. More importantly, within the gain scheduling control framework, the proposed approach can be easily extended to linear parameter-varying systems with saturated inputs and broaden the applicability of our method.

The main contribution of this article includes the following two aspects. First, a gain-scheduling output feedback controller is proposed to guarantee regional stability of the closed-loop system and provide disturbance/error attenuation measured in $\mathcal{L}_{2}$ gain. Our approach is based on the NDI (norm-bounded differential inclusion) model and is computationally more efficient than that of the PDI (polytopic differential inclusion) model. Specifically, the synthesis condition of PDI models involves a set of LMIs and the number of LMIs increases with the number of vertices. On the other hand, the synthesis condition of NDI models only has four matrix inequalities with much less optimization variables. Moreover, explicit formulas are derived to calculate controller gains, which could reduce the computational cost compared to the method of directly solving the LMI-based condition.

Second, our approach provides a unified framework of treating saturated/unsaturated control inputs, open-loop stable/unstable plants. More specifically, this problem formulation will fill the gap between the general $\mathcal{H}_{\infty}$ control theory for linear plants and the control method for linear plants with all input channels saturated, therefore it is of theoretical interest on its own. In addition, the study on the linear plants with partial control channels subject to saturation are also useful from engineering viewpoint. For multi-input systems, one may wonder which input channel saturation will have a stronger effect on the closed-loop performance. To answer this question, we can calculate the optimal performance of the closed-loop system with just one input channel saturated by using the results developed from this research and repeat the procedure for other input channels. By comparing the results from this trade study, proper selection of actuators can be made to reduce the cost of the control system. Although it is true that unsaturated input channels could be treated by assuming their saturation levels very large and using synthesis methods for linear plants with all input channels saturated. However, the results obtained in this way are not theoretically well justified.

The notations used in this paper are rather standard. $\mathbf{R}$ stands for the set of real numbers and $\mathbf{R}_{+}$ for the non-negative real numbers. $\mathbf{Z}_{+}$is the set of non-negative integers. $\mathbf{R}^{m \times n}$ is the set of real $m \times n$ matrices. We use $\mathbf{S}^{n \times n}$ to denote real, symmetric $n \times n$ matrices, and $\mathbf{S}_{+}^{n \times n}$ for positivedefinite matrices. The identity matrix of any dimension is denoted by $I$. For real matrices, the hermitian transpose $\operatorname{He}\{\cdot\}$ is defined as $\operatorname{He}\{M\}=M+M^{T}$. A block-diagonal matrix with matrices $X_{1}, X_{2}, \cdots, X_{p}$ on its main diagonal is denoted as diag $\left\{X_{1}, X_{2}, \cdots, X_{p}\right\}$. In large symmetric matrix expressions, terms denoted as $\star$ will be induced by symmetry. For two integers $k_{1}, k_{2}, k_{1}<k_{2}$, we denote $\mathbf{I}\left[k_{1}, k_{2}\right]=\left\{k_{1}, k_{1}+1, \cdots, k_{2}\right\}$. The space of square integrable functions is denoted by $\mathcal{L}_{2}$, that is, for any $x \in \mathcal{L}_{2}$

$$
\|x\|_{2}:=\left(\int_{0}^{\infty} x^{T}(t) x(t) d t\right)^{\frac{1}{2}}<\infty .
$$

$C o\{S\}$ denotes the convex hull of a set $S$.

This paper is organized as follows. In Section 2, we formulate the problem that will be addressed 
in our paper. The main synthesis condition and some remarks are given in Section 3. Then explicit formulae are derived in Section 4 to calculate the controller gains. In Section 5, numerical examples are provided to demonstrate our approach and detailed comparison is made between some existing methods and our approach. Finally, Section 6 concludes this paper.

\section{Problem formulation}

Consider the linear plant subject to partial actuator saturations,

$$
\left\{\begin{array}{l}
\dot{x}=A x+B_{1} d+B_{2 s} \operatorname{sat}\left(u_{s}\right)+B_{2 n} u_{n} \\
e=C_{1} x+D_{11} d+D_{12 s} \operatorname{sat}\left(u_{s}\right)+D_{12 n} u_{n} \\
y=C_{2} x+D_{21} d
\end{array}\right.
$$

where $x \in \mathbf{R}^{n_{x}}$ is the plant state; $u_{s} \in \mathbf{R}^{n_{s}}$ is the control input subject to saturation; $u_{n} \in$ $\mathbf{R}^{n_{n}}$ represent the control inputs which will not be saturated; $d \in \mathbf{R}^{n_{d}}$ is the exogenous input, possibly including disturbance, measurement noise or reference signals; $y \in \mathbf{R}^{n_{y}}$ is the measurement output and $e \in \mathbf{R}^{n_{e}}$ is the performance output. $\operatorname{sat}(\cdot)$ is a vector saturation function with the saturation levels given by a vector $\bar{u} \in \mathbf{R}^{n_{s}}, \bar{u}_{i}>0, i \in \mathbf{I}\left[1, n_{s}\right]$. More specifically, $\operatorname{sat}\left(u_{i}\right)=$ $\operatorname{sgn}\left(u_{i}\right) \min \left\{\bar{u}_{i},\left|u_{i}\right|\right\}$. Throughout this article, it is assumed that

(A1) $\left(A,\left[\begin{array}{ll}B_{2 s} & B_{2 n}\end{array}\right]\right)$ is stabilizable and $\left(C_{2}, A\right)$ is detectable,

(A2) $D_{21}$ has full row rank and $\left[\begin{array}{cc}I_{n_{s}} & 0 \\ D_{12 s} & D_{12 n}\end{array}\right]$ has full column rank.

The deadzone nonlinearity is closely related to saturation function by $\mathrm{dz}(u)=u-\operatorname{sat}(u)$. In global analysis, we often use

$$
\mathrm{dz}(u) \in \operatorname{sect}[0, I],
$$

where sect(.) means the nonlinear sector as defined in [13]. This relation holds for all $u \in \mathbf{R}^{n_{s}}$ but could be conservative over a local region where the system operates. On the other hand, the deadzone function can be sharpened for regional analysis of unstable plants as shown in the following lemma.

Lemma 1 ([11]) Let $h(x)=H x$ be a linear map and suppose $e_{i}^{T} H x \in\left[-\bar{u}_{i}, \bar{u}_{i}\right]$, where $e_{i}$ denotes the $i$ th column of the unity matrix. For any $u_{i}$, we have $\operatorname{sat}\left(u_{i}\right) \in \operatorname{Co}\left\{u_{i}, e_{i}^{T} H x\right\}$ and $\mathrm{dz}\left(u_{i}\right)=$ $\theta_{i}\left(u_{i}-e_{i}^{T} H x\right)$ for some $\theta_{i} \in[0,1]$.

Using the deadzone function, the state equation of the plant can be rewritten as

$$
\begin{aligned}
{\left[\begin{array}{c}
\dot{x} \\
u_{s} \\
e \\
y
\end{array}\right] } & =\left[\begin{array}{cccc}
A & -B_{2} E & B_{1} & B_{2} \\
0 & 0 & 0 & E^{T} \\
C_{1} & -D_{12} E & D_{11} & D_{12} \\
C_{2} & 0 & D_{21} & 0
\end{array}\right]\left[\begin{array}{l}
x \\
p \\
d \\
u
\end{array}\right] \\
p & =\mathrm{dz}\left(u_{s}\right),
\end{aligned}
$$

where $B_{2}=\left[\begin{array}{ll}B_{2 s} & B_{2 n}\end{array}\right], D_{12}=\left[\begin{array}{ll}D_{12 s} & D_{12 n}\end{array}\right]$ and $u=\left[\begin{array}{l}u_{s} \\ u_{n}\end{array}\right], E=\left[\begin{array}{c}I_{n_{s}} \\ 0\end{array}\right]$. 
For a matrix $\left[\begin{array}{ll}H_{1} & H_{2}\end{array}\right]$, we will define the set

$$
\mathcal{L}\left(\left[\begin{array}{ll}
H_{1} & H_{2}
\end{array}\right]\right)=\left\{\left[\begin{array}{c}
x \\
x_{k}
\end{array}\right] \in \mathbf{R}^{2 n_{x}}:\left|e_{j}^{T}\left[\begin{array}{ll}
H_{1} & H_{2}
\end{array}\right]\left[\begin{array}{c}
x \\
x_{k}
\end{array}\right]\right| \leq \bar{u}_{j}, j \in \mathbf{I}\left[1, n_{s}\right]\right\} .
$$

Again $e_{j}$ denotes the $j$ th column of the identity matrix. $x_{k}$ is the state of the controller to be designed. Then from Lemma 1, the nonlinear equation (3) can be replaced by

$$
p=\Theta\left(u_{s}-H_{1} x-H_{2} x_{k}\right), \quad \Theta=\left\{\operatorname{diag}\left\{\theta_{1}, \cdots, \theta_{n_{s}}\right\}: 0 \leq \theta_{j} \leq 1, j \in \mathbf{I}\left[1, n_{s}\right]\right\} .
$$

The gain-scheduling controller as described in eqns. (5)-(6) will be synthesized for the plant (1)

$$
\begin{aligned}
{\left[\begin{array}{c}
\dot{x}_{k} \\
q_{k} \\
u
\end{array}\right] } & =\left[\begin{array}{ccc}
A_{k} & B_{k 0} & B_{k 1} \\
C_{k 0} & D_{k 00} & D_{k 01} \\
C_{k 1} & D_{k 10} & D_{k 11}
\end{array}\right]\left[\begin{array}{c}
x_{k} \\
p_{k} \\
y
\end{array}\right] \\
p_{k} & =\Theta\left(q_{k}-H_{k 1} x-H_{k 2} x_{k}\right),
\end{aligned}
$$

where $x_{k}$ is the state of the controller. The number of controller states is chosen to be the same as that of plant states $n_{x} . p_{k}, q_{k} \in \mathbf{R}^{n_{s}}$ are the pseudo-input and output of the controller, respectively. It can be observed that there exists an algebraic loop between $p_{k}$ and $q_{k}$. But it will not cause any problem as long as $q_{k}$ can be resolved uniquely from equations (5)(6) by inverting matrix $\left(I-D_{k 00} \Theta\right)$. This is related to the well-posedness of the closed-loop system. In our approach, it can be solved as long as the synthesis condition in Theorem 1 is solvable.

According to eqn. (4), the diagonal elements of $\Theta$ can be calculated by (7) in real time.

$$
\theta_{j}=\left\{\begin{array}{ll}
0, & -\bar{u}_{s_{j}} \leq u_{s_{j}} \leq \bar{u}_{s_{j}} \\
\frac{\mathrm{dz}\left(u_{s_{j}}\right)}{u_{s_{j}}-e_{j}^{T}\left(H_{1} x+H_{2} x_{k}\right)}, & \text { else }
\end{array},\right.
$$

$j \in \mathbf{I}\left[1, n_{s}\right]$.

As indicated by eqn. (7), when $\left|u_{s_{j}}\right|>\bar{u}_{s_{j}}, \theta_{j}$ will be calculated by $\frac{\mathrm{dz}\left(u_{s_{j}}\right)}{u_{s_{j}}-e_{j}^{T}\left(H_{1} x+H_{2} x_{k}\right)}$. At this time, $\operatorname{dz}\left(u_{s_{j}}\right)=u_{s_{j}} \mp \bar{u}_{s_{j}}$, and $\left|e_{j}^{T}\left(H_{1} x+H_{2} x_{k}\right)\right| \leq \bar{u}_{s_{j}}$. For the case that $\left|e_{j}^{T}\left(H_{1} x+H_{2} x_{k}\right)\right|=\bar{u}_{s_{j}}, \theta_{j}=1$. While for the other case, $0<\theta_{j}<1$. So the denominator of eqn. (7) can never vanish because of $\left|u_{s_{j}}\right|>\bar{u}_{s_{j}}$. Consequently, in any cases $\theta_{j}$ will definitely be well defined and within the interval $[0,1]$.

As a result, the controller parameters will depend on $\Theta$ (i.e., the actuator saturation status) in the form of LFT. Note that this form of gain-scheduling controller is similar to the ones shown in [1] and [18]. But the measurable parameter is restricted to $0 \leq \Theta \leq I$ here.

It is observed from eqns. (6) and (7) that the state vector of the plant $x$ explicitly appears in the controller. If all the states of the plant are measurable, the parameter $\Theta$ can be computed in real time and the gain-scheduling controller can be implemented directly. However, for the output feedback control problem, the plant state information is generally not available. In this case we can assume that $H_{1}=H_{1 k}=0$. Then we can implement such an output controller. However in order to get a complete understanding of the role of $H_{1}$ and $H_{1 k}$, we still regard $H_{1}$ and $H_{k 1}$ as constant matrices in the following derivation.

In this research, our objective is to synthesize a gain-scheduling output feedback controller to stabilize linear systems with partial actuator saturation (1) and minimize its disturbance effect. 
For disturbance attenuation, we are mainly concerned with a class of energy-bounded disturbances

$$
\mathcal{W}_{s}=\left\{d: \mathbf{R}_{+} \rightarrow \mathbf{R}^{n_{d}}, \int_{0}^{\infty} d^{T}(\tau) d(\tau) d \tau \leq s^{2}, d \in \mathcal{L}_{2}\right\}
$$

The level of disturbance attenuation will be measured by the performance index

$$
\sup _{d \in \mathcal{W}_{s}, x(0)=0} \frac{\|e\|_{2}}{\|d\|_{2}}
$$

which specifies a regional $\mathcal{L}_{2}$ gain.

By combining the plant and the controller together, we obtain the closed-loop system as below:

$$
\begin{aligned}
{\left[\begin{array}{c}
\dot{x}_{c l} \\
q_{c l} \\
e
\end{array}\right] } & =\left[\begin{array}{ccc}
A_{c l} & B_{0, c l} & B_{1, c l} \\
C_{0, c l} & D_{00, c l} & D_{01, c l} \\
C_{1, c l} & D_{10, c l} & D_{11, c l}
\end{array}\right]\left[\begin{array}{c}
x_{c l} \\
p_{c l} \\
d
\end{array}\right] \\
p_{c l} & =\left[\begin{array}{cc}
\Theta & 0 \\
0 & \Theta
\end{array}\right]\left(q_{c l}-H x_{c l}\right),
\end{aligned}
$$

where $x_{c l}=\left[\begin{array}{c}x \\ x_{k}\end{array}\right], q_{c l}=\left[\begin{array}{c}u_{s} \\ q_{k}\end{array}\right], p_{c l}=\left[\begin{array}{c}p \\ p_{k}\end{array}\right]$, and

$$
\begin{aligned}
& {\left[\begin{array}{ccc}
A_{c l} & B_{0, c l} & B_{1, c l} \\
C_{0, c l} & D_{00, c l} & D_{01, c l} \\
C_{1, c l} & D_{10, c l} & D_{11, c l}
\end{array}\right]} \\
& =\left[\begin{array}{cc|cc|c}
A & 0 & -B_{2} E & 0 & B_{1} \\
0 & 0 & 0 & 0 & 0 \\
\hline 0 & 0 & 0 & 0 & 0 \\
0 & 0 & 0 & 0 & 0 \\
\hline C_{1} & 0 & -D_{12} E & 0 & D_{11}
\end{array}\right]+\left[\begin{array}{ccc}
0 & 0 & B_{2} \\
I & 0 & 0 \\
\hline 0 & 0 & E^{T} \\
0 & I & 0 \\
\hline 0 & 0 & D_{12}
\end{array}\right]\left[\begin{array}{ccc}
A_{k} & B_{k 0} & B_{k 1} \\
C_{k 0} & D_{k 00} & D_{k 01} \\
C_{k 1} & D_{k 10} & D_{k 11}
\end{array}\right]\left[\begin{array}{cc|cc|c}
0 & I & 0 & 0 & 0 \\
0 & 0 & 0 & I & 0 \\
C_{2} & 0 & 0 & 0 & D_{21}
\end{array}\right] \\
& :=\left[\begin{array}{ccc}
\mathcal{A}_{0} & \mathcal{B}_{01} & \mathcal{B}_{02} \\
\mathcal{C}_{01} & \mathcal{D}_{011} & \mathcal{D}_{012} \\
\mathcal{C}_{02} & \mathcal{D}_{021} & \mathcal{D}_{022}
\end{array}\right]+\left[\begin{array}{c}
\mathcal{E}_{0} \\
\mathcal{E}_{1} \\
\mathcal{E}_{2}
\end{array}\right]\left[\begin{array}{ccc}
A_{k} & B_{k 0} & B_{k 1} \\
C_{k 0} & D_{k 00} & D_{k 01} \\
C_{k 1} & D_{k 10} & D_{k 11}
\end{array}\right]\left[\begin{array}{lll}
\mathcal{G}_{0} & \mathcal{G}_{1} & \mathcal{G}_{2}
\end{array}\right] \\
& H=\left[\begin{array}{cc}
H_{1} & H_{2} \\
H_{k 1} & H_{k 2}
\end{array}\right] \text {. }
\end{aligned}
$$

The above system description is valid for the partially saturated system when $\left[\begin{array}{c}x \\ x_{k}\end{array}\right] \in \mathcal{L}\left(\left[\begin{array}{ll}H_{1} & H_{2}\end{array}\right]\right)$.

\section{Gain-Scheduling Control with Partial Actuator Saturation}

Motivated by the general results in both $\mathcal{H}_{\infty}$ and gain-scheduling control theories [6] [18], the main result of this research will be presented in the following theorem. 
Theorem 1 Given scalars $s>0$ and $\gamma>0$, if there exist positive definite matrices $R, S \in \mathbf{S}_{+}^{n}$, diagonal matrices $L, J>0$ and matrixes $\hat{H}_{1}, \hat{H}_{2}, \hat{H}_{1 k} \in R^{n_{s} \times n}$ such that

$$
\begin{aligned}
& {\left[\begin{array}{cc}
\mathcal{N}_{\tilde{\Phi}}^{T} & 0 \\
0 & I
\end{array}\right]\left[\begin{array}{ccc|c}
R A^{T}+A R & -B_{2} E L-\hat{H}_{2}^{T} & R C_{1}^{T} & B_{1} \\
-L E^{T} B_{2}^{T}-\hat{H}_{2} & -2 L & -L E^{T} D_{12}^{T} & 0 \\
C_{1} R & -D_{12} E L & -\gamma^{2} I & D_{11} \\
\hline B_{1}^{T} & 0 & D_{11}^{T} & -I
\end{array}\right]\left[\begin{array}{cc}
\mathcal{N}_{\tilde{\Phi}} & 0 \\
0 & I
\end{array}\right]<0} \\
& {\left[\begin{array}{cc}
\mathcal{N}_{\Gamma}^{T} & 0 \\
0 & I
\end{array}\right]\left[\begin{array}{cc|cc}
A^{T} S+S A & S B_{1} & -S B_{2} E-\hat{H}_{1 k}^{T} & C_{1}^{T} \\
B_{1}^{T} S & -I & 0 & D_{11}^{T} \\
\hline-E^{T} B_{2}^{T} S-\hat{H}_{1 k} & 0 & -2 J & -E^{T} D_{12}^{T} \\
C_{1} & D_{11} & -D_{12} E & -\gamma^{2} I
\end{array}\right]\left[\begin{array}{cc}
\mathcal{N}_{\Gamma} & 0 \\
0 & I
\end{array}\right]<0} \\
& {\left[\begin{array}{cc}
R & I \\
I & S
\end{array}\right]>0} \\
& {\left[\begin{array}{ll}
L & I \\
I & J
\end{array}\right]>0} \\
& {\left[\begin{array}{ccc}
\frac{\bar{u}_{\ell}^{2}}{s^{2}} & e_{\ell}^{T} \hat{H}_{2} & e_{\ell}^{T} \hat{H}_{1} \\
\hat{H}_{2}^{T} e_{\ell} & R & I \\
\hat{H}_{1}^{T} e_{\ell} & I & S
\end{array}\right] \geq 0, \quad \forall \ell \in \mathbf{I}\left[1, n_{s}\right]}
\end{aligned}
$$

where $\mathcal{N}_{\tilde{\Phi}}:=\left[\begin{array}{lll}\mathcal{N}_{\tilde{\Phi}_{1}}^{T} & \mathcal{N}_{\tilde{\Phi}_{2}}^{T} & \mathcal{N}_{\tilde{\Phi}_{3}}^{T}\end{array}\right]^{T}$ and $\mathcal{N}_{\Gamma}:=\left[\begin{array}{ll}\mathcal{N}_{\Gamma_{1}}^{T} & \mathcal{N}_{\Gamma_{2}}^{T}\end{array}\right]^{T}$ denote matrices whose columns form bases of $\operatorname{Ker}\left[\begin{array}{lll}B_{2}^{T} & E & D_{12}^{T}\end{array}\right]$ and $\operatorname{Ker}\left[\begin{array}{ll}C_{2} & D_{21}\end{array}\right]$ respectively, and

$$
\left[\begin{array}{cc}
\hat{H}_{1} & \hat{H}_{2} \\
\hat{H}_{1 k} & \hat{H}_{2 k}
\end{array}\right]=\left[\begin{array}{cc}
I & 0 \\
J & V
\end{array}\right]\left[\begin{array}{cc}
H_{1} & H_{2} \\
H_{1 k} & H_{2 k}
\end{array}\right]\left[\begin{array}{cc}
I & R \\
0 & M^{T}
\end{array}\right]
$$

with $M N^{T}=I-R S, U V^{T}=I-L J$, then a nth-order gain-scheduling controller in the form of (5)-(6) will locally asymptotically stabilize the plant (1) and render the $\mathcal{L}_{2}$ gain of the closed loop system less than $\gamma$ for any bounded disturbance $d \in \mathcal{W}_{s}$.

Proof: Use a quadratic Lyapunov function $V\left(x_{c l}\right)=x_{c l}^{T} P x_{c l}$ with $P>0$ and a positive-definite matrix $\Lambda=\left[\begin{array}{ll}\Lambda_{1} & \Lambda_{2} \\ \Lambda_{2} & \Lambda_{3}\end{array}\right]>0$ commutable with $\operatorname{diag}\{\Theta, \Theta\}$, i.e.,

$$
\Lambda \operatorname{diag}\{\Theta, \Theta\}=\operatorname{diag}\{\Theta, \Theta\} \Lambda .
$$

It is clear that $\Lambda_{1}, \Lambda_{2}$ and $\Lambda_{3}$ should be diagonal matrices. Then a regional performance condition

$$
\dot{V}+\frac{1}{\gamma^{2}} e^{T} e-d^{T} d+p_{c l}^{T} \Lambda\left(q_{c l}-H x_{c l}-p_{c l}\right)+\left(q_{c l}-H x_{c l}-p_{c l}\right)^{T} \Lambda p_{c l}<0
$$

and the set inclusion condition

$$
\left\{x_{c l}: x_{c l}^{T} P x_{c l} \leq s^{2}\right\} \subset \mathcal{L}\left(\left[\begin{array}{ll}
H_{1} & H_{2}
\end{array}\right]\right)
$$

will guarantee the closed-loop stability and $\mathcal{L}_{2}$ gain performance for partially saturated linear systems.

According to the robust control theory, the first three terms relate to the $\mathcal{L}_{2}$ performance of the closed-loop system and the rest of (17) correspond to the sector constraint on the matrix 
$\operatorname{diag}\{\Theta, \Theta\}$. By using the $\mathcal{S}$-procedure, we get the regional performance condition (17). The set inclusion condition (18) ensures validity of the linear systems description.

Using the scaled Bounded Real Lemma and exploring the set inclusion relation [4], we obtain the following two equations for the regional performance condition and the set inclusion condition.

$$
\begin{aligned}
& {\left[\begin{array}{cccc}
A_{c l}^{T} P+P A_{c l} & \star & \star & \star \\
B_{0, c l}^{T} P+\Lambda\left(C_{0, c l}-H\right) & \Lambda\left(D_{00, c l}-I\right)+\left(D_{00, c l}-I\right)^{T} \Lambda & \star & \star \\
B_{1, c l}^{T} P & D_{01, c l}^{T} \Lambda & -I & \star \\
C_{1, c l} & D_{10, c l} & D_{11, c l} & -\gamma^{2} I
\end{array}\right]<0} \\
& {\left[\begin{array}{cc}
\frac{\bar{u}_{\ell}^{2}}{s^{2}} & e_{\ell}^{T}\left[\begin{array}{ll}
H_{1} & H_{2}
\end{array}\right] \\
{\left[\begin{array}{c}
H_{1}^{T} \\
H_{2}^{T}
\end{array}\right] e_{\ell}} & P
\end{array}\right] \geq 0,} \\
&
\end{aligned}
$$

In the following, we will prove that (10)-(14) is equivalent to the regional performance condition and the set inclusion condition.

Taking eqn. (8) into consideration, the inequality (19) can be rewritten as

$$
\Psi+\Gamma^{T} \Pi^{T} \Phi+\Phi^{T} \Pi \Gamma<0,
$$

where

$$
\begin{aligned}
\Psi & :=\left[\begin{array}{cccc}
\mathcal{A}_{0}^{T} P+P \mathcal{A}_{0} & \star & \star & \star \\
\mathcal{B}_{01}^{T} P+\Lambda\left(\mathcal{C}_{01}-H\right) & \Lambda\left(\mathcal{D}_{011}-I\right)+\left(\mathcal{D}_{011}-I\right)^{T} \Lambda & \star & \star \\
\mathcal{B}_{02}^{T} P & \mathcal{D}_{012}^{T} \Lambda & -I & \star \\
\mathcal{C}_{02} & D_{021} & \mathcal{D}_{022} & -\gamma^{2} I
\end{array}\right] \\
\Pi & :=\left[\begin{array}{ccc}
A_{k} & B_{k 0} & B_{k 1} \\
C_{k 0} & D_{k 00} & D_{k 01} \\
C_{k 1} & D_{k 10} & D_{k 11}
\end{array}\right] \\
\Gamma & :=\left[\begin{array}{llll}
\mathcal{G}_{0} & \mathcal{G}_{1} & \mathcal{G}_{2} & 0
\end{array}\right]=\left[\begin{array}{cc|cc|c|c}
0 & I & 0 & 0 & 0 & 0 \\
0 & 0 & 0 & I & 0 & 0 \\
C_{2} & 0 & 0 & 0 & D_{21} & 0
\end{array}\right] \\
\Phi & :=\left[\begin{array}{llll|ll|l|l}
\mathcal{E}_{0}^{T} P & \mathcal{E}_{1}^{T} \Lambda & 0 & \mathcal{E}_{2}^{T}
\end{array}\right]=\left[\begin{array}{cc|cc|c|c}
0 & I & 0 & 0 & 0 \\
0 & 0 & 0 & I & 0 & 0 \\
B_{2}^{T} & 0 & E & 0 & 0 & D_{12}^{T}
\end{array}\right]\left[\begin{array}{cccc}
P & 0 & 0 & 0 \\
0 & \Lambda & 0 & 0 \\
0 & 0 & I & 0 \\
0 & 0 & 0 & I
\end{array}\right] .
\end{aligned}
$$

Applying Elimination Lemma [18], eqn. (21) is equivalent to

$$
\left\{\begin{array}{l}
\mathcal{H}_{\Phi}^{T} \Psi \mathcal{H}_{\Phi}<0 \\
\mathcal{H}_{\Gamma}^{T} \Psi \mathcal{H}_{\Gamma}<0
\end{array}\right.
$$

where $\mathcal{H}_{\Gamma}$ and $\mathcal{H}_{\Phi}$ are the null matrices of $\Gamma$ and $\Phi$, respectively. Note that $\Phi=\tilde{\Phi}\left[\begin{array}{cccc}P & 0 & 0 & 0 \\ 0 & \Lambda & 0 & 0 \\ 0 & 0 & I & 0 \\ 0 & 0 & 0 & I\end{array}\right]$ with $\tilde{\Phi}=\left[\begin{array}{cccc}\mathcal{E}_{0}^{T} & \mathcal{E}_{1}^{T} & 0 & \mathcal{E}_{2}^{T}\end{array}\right]$. Therefore, eqn. (22) can be transformed to

$$
\left\{\begin{array}{l}
\mathcal{H}_{\tilde{\Phi}}^{T} \tilde{\Psi} \mathcal{H}_{\tilde{\Phi}}<0 \\
\mathcal{H}_{\Gamma}^{T} \Psi \mathcal{H}_{\Gamma}<0
\end{array}\right.
$$


where $\mathcal{H}_{\tilde{\Phi}}$ is the null matrix of $\tilde{\Phi}$ and

$$
\tilde{\Psi}=\left[\begin{array}{cccc}
P^{-1} \mathcal{A}_{0}^{T}+\mathcal{A}_{0} P^{-1} & \star & \star & \star \\
\Lambda^{-1} \mathcal{B}_{01}^{T}+\left(\mathcal{C}_{01}-H\right) P^{-1} & \left(\mathcal{D}_{011}-I\right) \Lambda^{-1}+\Lambda^{-1}\left(\mathcal{D}_{011}-I\right)^{T} & \star & \star \\
\mathcal{B}_{02}^{T} & \mathcal{D}_{012}^{T} & -I & \star \\
\mathcal{C}_{02} P^{-1} & \mathcal{D}_{021} \Lambda^{-1} & \mathcal{D}_{022} & -\gamma^{2} I
\end{array}\right] .
$$

We partition Lyapunov matrix $P$ and scaling matrix $\Lambda$ according to plant and controller states as

$$
\begin{aligned}
P & =\left[\begin{array}{cc}
S & N \\
N^{T} & \star_{1}
\end{array}\right], & P^{-1}=\left[\begin{array}{cc}
R & M \\
M^{T} & \star_{2}
\end{array}\right], \\
\Lambda & =\left[\begin{array}{cc}
J & V \\
V^{T} & \star_{3}
\end{array}\right], & \Lambda^{-1}=\left[\begin{array}{cc}
L & U \\
U^{T} & \star_{4}
\end{array}\right] .
\end{aligned}
$$

Since $\mathcal{H}_{\tilde{\Phi}}=\left[\begin{array}{cccccc}\mathcal{N}_{\tilde{\Phi}_{1}}^{T} & 0 & \mathcal{N}_{\tilde{\Phi}_{2}}^{T} & 0 & 0 & \mathcal{N}_{\tilde{\Phi}_{3}}^{T} \\ 0 & 0 & 0 & 0 & I & 0\end{array}\right]^{T}$, we obtain from $\mathcal{H}_{\tilde{\Phi}}^{T} \tilde{\Psi} \mathcal{H}_{\tilde{\Phi}}<0$ that

$$
\left[\begin{array}{cccc}
\mathcal{N}_{\tilde{\Phi}_{1}}^{T} & \mathcal{N}_{\tilde{\Phi}_{2}}^{T} & 0 & \mathcal{N}_{\tilde{\Phi}_{3}}^{T} \\
0 & 0 & I & 0
\end{array}\right]\left[\begin{array}{cccc}
R A^{T}+A R & -B_{2} E L-\hat{H}_{2}^{T} & B_{1} & R C_{1}^{T} \\
-L E^{T} B_{2}^{T}-\hat{H}_{2} & -2 L & 0 & -L E^{T} D_{12}^{T} \\
B_{1}^{T} & 0 & -I & D_{11}^{T} \\
C_{1} R & -D_{12} E L & D_{11} & -\gamma^{2} I
\end{array}\right]\left[\begin{array}{cc}
\mathcal{N}_{\tilde{\Phi}_{1}} & 0 \\
\mathcal{N}_{\tilde{\Phi}_{2}} & 0 \\
0 & I \\
\mathcal{N}_{\tilde{\Phi}_{3}} & 0
\end{array}\right]<0 .
$$

By swapping the last two rows and columns, we get

$$
\left[\begin{array}{cccc}
\mathcal{N}_{\tilde{\Phi}_{1}}^{T} & \mathcal{N}_{\tilde{\Phi}_{2}}^{T} & \mathcal{N}_{\tilde{\Phi}_{3}}^{T} & 0 \\
0 & 0 & 0 & I
\end{array}\right]\left[\begin{array}{cccc}
R A^{T}+A R & -B_{2} E L-\hat{H}_{2}^{T} & R C_{1}^{T} & B_{1} \\
-L E^{T} B_{2}^{T}-\hat{H}_{2} & -2 L & -L E^{T} D_{12}^{T} & 0 \\
C_{1} R & -D_{12} E L & -\gamma^{2} I & D_{11} \\
B_{1}^{T} & 0 & D_{11}^{T} & -I
\end{array}\right]\left[\begin{array}{cc}
\mathcal{N}_{\tilde{\Phi}_{1}} & 0 \\
\mathcal{N}_{\tilde{\Phi}_{2}} & 0 \\
\mathcal{N}_{\tilde{\Phi}_{3}} & 0 \\
0 & I
\end{array}\right]<0
$$

which is the same as (10).

Similarly, $\mathcal{H}_{\Gamma}=\left[\begin{array}{cccccc}\mathcal{N}_{\Gamma_{1}}^{T} & 0 & 0 & 0 & \mathcal{N}_{\Gamma_{2}}^{T} & 0 \\ 0 & 0 & I & 0 & 0 & 0 \\ 0 & 0 & 0 & 0 & 0 & I\end{array}\right]^{T}$, we have from $\mathcal{H}_{\Gamma}^{T} \Psi \mathcal{H}_{\Gamma}<0$ that

$$
\left[\begin{array}{cccc}
\mathcal{N}_{\Gamma_{1}}^{T} & \mathcal{N}_{\Gamma_{2}}^{T} & 0 & 0 \\
0 & 0 & I & 0 \\
0 & 0 & 0 & I
\end{array}\right]\left[\begin{array}{cccc}
A^{T} S+S A & S B_{1} & -S B_{2} E-\hat{H}_{1 k}^{T} & C_{1}^{T} \\
B_{1}^{T} S & -I & 0 & D_{11}^{T} \\
-E^{T} B_{2}^{T} S-\hat{H}_{1 k} & 0 & -2 J & -E^{T} D_{12}^{T} \\
C_{1} & D_{11} & -D_{12} E & -\gamma^{2} I
\end{array}\right]\left[\begin{array}{ccc}
\mathcal{N}_{\Gamma_{1}} & 0 & 0 \\
\mathcal{N}_{\Gamma_{2}} & 0 & 0 \\
0 & I & 0 \\
0 & 0 & I
\end{array}\right]<0 .
$$

This confirms equation (11).

As for the eqns. (12)-(13), according to [18], $P>0, P^{-1}>0$ if and only if eqn. (12) holds. Similarly, $\Lambda>0$, and $\Lambda^{-1}>0$ is equivalent to condition (13).

Now, we have proven that eqns. (10)-(13) is equivalent to the condition (22), therefore guarantees the condition (19). 
To verify eqn. (14), we choose the invertible matrix $Z=\operatorname{diag}\left\{1,\left[\begin{array}{cc}R & M \\ I & 0\end{array}\right]\right\}$ and multiply condition (20) by $Z$ from the left and $Z^{T}$ from the right side

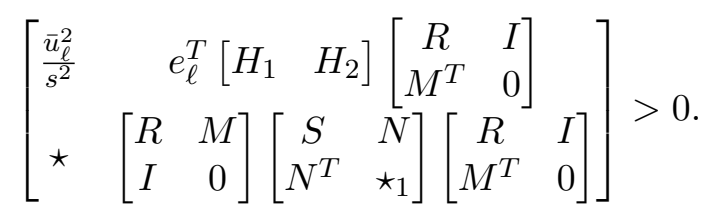

Using the fact $\left[\begin{array}{cc}S & N \\ N^{T} & \star_{1}\end{array}\right]\left[\begin{array}{cc}R & M \\ M^{T} & \star_{2}\end{array}\right]=I$, we get

$$
\left[\begin{array}{ccc}
\bar{u}_{\ell}^{2} & e_{\ell}^{T}\left(H_{1} R+H_{2} M^{T}\right) & e_{\ell}^{T} H_{1} \\
\star & R & I \\
\star & \star & S
\end{array}\right]>0 \quad \forall \ell \in \mathbf{I}\left[1, n_{s}\right] .
$$

Then condition (14) is confirmed through variable changes (15). So (14) will guarantee the set inclusion condition.

Q.E.D.

Remark 1 Note that Theorem 1 provides a feasible condition for the existence of the gain-scheduling controller in the form of (5)-(6) which depends on both $x$ and $x_{k}$. To get a practical output feedback controller, we need to set $\hat{H}_{1}=\hat{H}_{1 k}=0$ in the control synthesis conditions (10)-(14) which corresponds to $H_{1}=H_{k}=0$ as mentioned before.

Remark 2 The synthesis conditions (10)-(14) are in the form of LMIs and can be solved efficiently using interior point optimization algorithms. Moreover, the feasibility condition (10)-(14) can be formulated as the following optimization problem

$$
\begin{aligned}
& \min _{R, S, L, J, \hat{H}_{1}, \hat{H}_{2}, \hat{H}_{1 k}} \gamma^{2} \\
& \text { subj. to }(10)-(14)
\end{aligned}
$$

to minimize the closed-loop performance $\gamma$. Moreover, it is noted that synthesis conditions do not depend explicitly on $\hat{H}_{2 k}$. This implies if the LMIs (10)-(14) are feasible, one could construct a gain-scheduling controller with any value $\hat{H}_{2 k}$. Nevertheless, the choice of $\hat{H}_{2 k}$ will affect resulting gain-scheduling controller gains. Therefore, one may use matrix $\hat{H}_{2 k}$ as a tuning parameter to help choosing a better controller.

Remark 3 Theorem 1 provides a gain-scheduling control synthesis condition in solving actuator saturation control problems in $\mathcal{H}_{\infty}$ context. With the introduction of matrix $E$, it bridges seamlessly between saturation control of linear systems and the standard $\mathcal{H}_{\infty}$ control problem. By choosing $E$ to be zero, it means all the input channels will not get saturated. Then the discussed problem degenerates into the standard $\mathcal{H}_{\infty}$ problem and our results should recover $\mathcal{H}_{\infty}$ synthesis condition in [6]. This can be observed by merely replacing $E, \hat{H}_{1}, \hat{H}_{2}, \hat{H}_{1 k}, \hat{H}_{2 k}, J$ and $L$ all by zeros in (10-12) and by removing eqns. (13) and (14). On the other hand, if $E$ becomes an identity matrix $I$, our result is directly applicable to the linear plant with all its input channels subject to actuator saturation. 
As a special case, if the open-loop system is asymptotically stable, the deadzone nonlinearity will be captured globally by the condition $\mathrm{dz}(u)=\Theta u, 0 \leq \Theta \leq I$. Then the equivalent open-loop system becomes

$$
\begin{aligned}
{\left[\begin{array}{c}
\dot{x} \\
u_{s} \\
e \\
y
\end{array}\right] } & =\left[\begin{array}{cccc}
A & -B_{2} E & B_{1} & B_{2} \\
0 & 0 & 0 & E^{T} \\
C_{1} & -D_{12} E & D_{11} & D_{12} \\
C_{2} & 0 & D_{21} & 0
\end{array}\right]\left[\begin{array}{l}
x \\
p \\
d \\
u
\end{array}\right] \\
p & =\Theta u_{s} .
\end{aligned}
$$

In other words, auxiliary linear subspace is not required in the equivalent LFT description. Then the gain-scheduled control synthesis condition would degenerate to eqns. (10)-(13) with $\hat{H}_{1}=\hat{H}_{2}=0$ and $\hat{H}_{1 k}=0$. This will be summarized in the following corollary.

Corollary 1 For an asymptotically stable open-loop system (1), given $s, \gamma>0$, if there exist positive definite matrices $R, S \in \mathbf{S}_{+}^{n}$, diagonal matrices $L, J>0$ such that eqns. (10)-(13) hold with $\hat{H}_{1}=\hat{H}_{2}=0$ and $\hat{H}_{1 k}=0$, then a nth-order gain-scheduling controller in the form of (5)-(6) with $H_{k 1}=H_{k 2}=0$ will globally asymptotically stabilize the plant (1) and render the $\mathcal{L}_{2}$ gain of the closed loop system less than $\gamma$ for any bounded disturbance $d \in \mathcal{W}_{s}$.

Remark 4 For open-unstable plants, it is of great significance to study the domain of attraction from the viewpoints of both theory and practice. On this regard, by assuming the exogenous input d to be 0 , we can study the domain of attraction of the closed-loop gain-scheduling system. Moreover, an output feedback gain-scheduling controller can be synthesized to stabilize the plant (1) at the origin with the maximized domain of attraction. This fact can be summarized in the following theorem.

Theorem 2 If there exist positive definite matrices $R, S \in \mathbf{S}_{+}^{n}$, diagonal matrices $L, J>0$ and matrixes $\hat{H}_{1}, \hat{H}_{2}, \hat{H}_{1 k} \in R^{n_{s} \times n}$ such that (12) (13) and the following LMIs hold

$$
\begin{aligned}
& \mathcal{N}_{\tilde{\Phi}_{D}}^{T}\left[\begin{array}{cc}
R A^{T}+A R & -B_{2} E L-\hat{H}_{2}^{T} \\
-L E^{T} B_{2}^{T}-\hat{H}_{2} & -2 L
\end{array}\right] \mathcal{N}_{\tilde{\Phi}_{D}}<0 \\
& {\left[\begin{array}{cr}
\mathcal{N}_{\Gamma_{D}}^{T} & 0 \\
0 & I
\end{array}\right]\left[\begin{array}{cc}
A^{T} S+S A & -S B_{2} E-\hat{H}_{1 k}^{T} \\
-E^{T} B_{2}^{T} S-\hat{H}_{1 k} & -2 J
\end{array}\right]\left[\begin{array}{cc}
\mathcal{N}_{\Gamma_{D}} & 0 \\
0 & I
\end{array}\right]<0} \\
& {\left[\begin{array}{ccc}
\bar{u}_{\ell}^{2} & e_{\ell}^{T} \hat{H}_{2} & e_{\ell}^{T} \hat{H}_{1} \\
\hat{H}_{2}^{T} e_{\ell} & R & I \\
\hat{H}_{1}^{T} e_{\ell} & I & S
\end{array}\right] \geq 0, \quad \forall \ell \in \mathbf{I}\left[1, n_{s}\right]}
\end{aligned}
$$

where $\mathcal{N}_{\tilde{\Phi}_{D}}:=\left[\begin{array}{ll}\mathcal{N}_{\tilde{\Phi}_{1}}^{T} & \mathcal{N}_{\tilde{\Phi}_{2}}^{T}\end{array}\right]^{T}$ and $\mathcal{N}_{\Gamma_{D}}:=\left[\mathcal{N}_{\Gamma_{1}}^{T}\right]^{T}$ denote matrices whose columns form bases of $\operatorname{Ker}\left[\begin{array}{ll}B_{2}^{T} & E\end{array}\right]$ and $\operatorname{Ker}\left[C_{2}\right]$ respectively, and then a nth-order gain-scheduling controller in the form of (5)-(6) will locally asymptotically stabilize the plant (1) at the origin with the ellipsoid of the form

$$
\Omega(P, 1)=x \in R^{n_{x}+n_{x}}: x^{T} P x \leq 1, P \in S_{+}^{\left(n_{c}+n_{c}\right) \times\left(n_{c}+n_{c}\right)}
$$

contained in the domain of attraction. 
Then with respect to a given shape reference set $\Omega\left(X_{R}, 1\right), X_{R} \subset S_{+}^{n_{c} \times n_{c}}$, by maximizing $\alpha>0$ such that

$$
\left.\alpha \Omega\left(X_{R}, 1\right) \subset \Omega(P, 1)\right|_{\left(x_{p}, 0\right)},
$$

which can be converted into an LMI constraint

$$
\frac{1}{\alpha^{2}} X_{R}-S \geq 0
$$

Then we can maximize the domain of attraction by solving an LMI optimization problem:

$$
\begin{array}{cc}
\max & \alpha \\
\text { subj. to } & (12-13)(26-28) \\
\text { and }(31) .
\end{array}
$$

Proof: Since the proof for this theorem is quite similar to the one for the main theorem, it is omitted here.

Q.E.D.

\section{Controller Reconstruction}

After solving the control synthesis condition in Theorem 1, it is necessary to reconstruct controller gains. In this section, explicit formulas for the gain-scheduling controllers will be derived through congruent transformation. Define the following matrixes

$$
\begin{array}{rlrl}
Z_{1} & =\left[\begin{array}{cc}
R & I \\
M^{T} & 0
\end{array}\right], & Z_{2}=\left[\begin{array}{cc}
I & S \\
0 & N^{T}
\end{array}\right], \\
T_{1}=\left[\begin{array}{cc}
L & I \\
U^{T} & 0
\end{array}\right], & T_{2}=\left[\begin{array}{cc}
I & J \\
0 & V^{T}
\end{array}\right] .
\end{array}
$$

Consequently, the Laypunov matrix $P$ and scaling matrix $\Lambda$ are given by

$$
P=Z_{2} Z_{1}^{-1} \quad \Lambda=T_{2} T_{1}^{-1} .
$$


Pre and post-multiplying condition (21) by $\operatorname{diag}\left\{Z_{1}^{T}, T_{1}^{T}, I, I\right\}$ and $\operatorname{diag}\left\{Z_{1}, T_{1}, I, I\right\}$ respectively, we obtain

$$
\begin{aligned}
& {\left[\begin{array}{c|c}
\left\{\begin{array}{c}
A R+R A^{T} \\
+B_{2} \hat{C}_{k 1}+\hat{C}_{k 1}^{T} B_{2}^{T}
\end{array}\right\} & \star \\
\hline A^{T}+\hat{A}_{k}+C_{2}^{T} \hat{D}_{k 11}^{T} B_{2}^{T} & \left\{\begin{array}{c}
A^{T} S+S A \\
+C_{2}^{T} \hat{B}_{k 1}^{T}+\hat{B}_{k 1} C_{2}
\end{array}\right\} \\
\hline\left\{\begin{array}{c}
-L E^{T} B_{2}^{T}+\hat{D}_{k 10}^{T} B_{2}^{T} \\
+E^{T} \hat{C}_{k 1}-\hat{H}_{2}
\end{array}\right\} & \hat{B}_{k 0}^{T}+E^{T} \hat{D}_{k 11} C_{2}-\hat{H}_{1} \\
-E^{T} B_{2}^{T}+\hat{C}_{k 0}-\hat{H}_{2 k} & -E^{T} B_{2}^{T} S+\hat{D}_{k 01} C_{2}-\hat{H}_{1 k} \\
B_{1}^{T}+D_{21}^{T} \hat{D}_{k 11}^{T} B_{2}^{T} & B_{1}^{T} S+D_{21}^{T} \hat{B}_{k 1}^{T} \\
C_{1} R+D_{12} \hat{C}_{k 1} & C_{1}+D_{12} \hat{D}_{k 11} C_{2}
\end{array}\right.} \\
& \left.\begin{array}{cccc}
\star & \star & \star & \star \\
\hline \star & \star & \star & \star \\
\hline E^{T} \hat{D}_{k 10}+\hat{D}_{k 10}^{T} E-2 L & \star & \star & \star \\
\hat{D}_{k 00}-2 I & -2 J & \star & \star \\
D_{21}^{T} \hat{D}_{k 11}^{T} E & D_{21}^{T} \hat{D}_{k 01}^{T} & -I & \star \\
-D_{12} E L+D_{12} \hat{D}_{k 10} & -D_{12} E & D_{11}+D_{12} \hat{D}_{k 11} D_{21} & -\gamma^{2} I
\end{array}\right] \\
& :=\left[\begin{array}{ccc}
\Sigma_{11} & \Sigma_{21}^{T} & \Omega_{1} \\
\Sigma_{21} & \Sigma_{22} & \Omega_{2} \\
\Omega_{1}^{T} & \Omega_{2}^{T} & \Delta
\end{array}\right]<0,
\end{aligned}
$$

in which the transformed controller gains and linear subspace matrices are defined as

$$
\begin{aligned}
{\left[\begin{array}{ccc}
\hat{A}_{k} & \hat{B}_{k 0} & \hat{B}_{k 1} \\
\hat{C}_{k 0} & \hat{D}_{k 00} & \hat{D}_{k 01} \\
\hat{C}_{k 1} & \hat{D}_{k 10} & \hat{D}_{k 11}
\end{array}\right]=} & {\left[\begin{array}{ccc}
N & 0 & S B_{2} \\
0 & V & J E^{T} \\
0 & 0 & I
\end{array}\right]\left[\begin{array}{ccc}
A_{k} & B_{k 0} & B_{k 1} \\
C_{k 0} & D_{k 00} & D_{k 01} \\
C_{k 1} & D_{k 10} & D_{k 11}
\end{array}\right]\left[\begin{array}{ccc}
M^{T} & 0 & 0 \\
0 & U^{T} & 0 \\
C_{2} R & 0 & I
\end{array}\right] } \\
& +\left[\begin{array}{ccc}
S A R & -S B_{2} E L & 0 \\
0 & 0 & 0 \\
0 & 0 & 0
\end{array}\right] .
\end{aligned}
$$

Note that condition (32) can be used to solve the proposed gain-scheduled saturation control problem through LMI optimization. However, the inequality has many optimization variables $\left(\hat{A}_{k}, \hat{B}_{k 0}, \hat{C}_{k 0}\right.$, etc.) involved and it is computationally expensive to solve it directly especially when the order of the plant is very high.

By Schur complement, condition (32) can be rewritten as

$$
\left[\begin{array}{cc}
\Sigma_{11}-\Omega_{1} \Delta^{-1} \Omega_{1}^{T} & \star \\
\Sigma_{21}-\Omega_{2} \Delta^{-1} \Omega_{1}^{T} & \Sigma_{22}-\Omega_{2} \Delta^{-1} \Omega_{2}^{T}
\end{array}\right]<0
$$

It is observed from the above equation that the $(1,1)$ block depends only on $\hat{C}_{k 0}$ and $\hat{C}_{k 1}$; the $(2,2)$ block depends only on $\hat{B}_{k 0}$ and $\hat{B}_{k 1}$; while only the off-diagonal block depends on $\hat{A}_{k}$. Therefore, solutions in explicit formulas for $\left[\begin{array}{ll}\hat{B}_{k 0} & \hat{B}_{k 1}\end{array}\right]$ and $\left[\begin{array}{lll}\hat{C}_{k 0}^{T} & \hat{C}_{k 1}\end{array}\right]$ can be obtained by a similar procedure as in [7] and will be summarized in Theorem 3 . 
Theorem 3 Given $R, S, \hat{H}_{1}, \hat{H}_{2}$, and $\hat{H}_{1 k}$ satisfying eqns. (10)-(14) and let $\hat{D}_{k 00}, \hat{D}_{k 01}, \hat{D}_{k 10}, \hat{D}_{k 11}$ be a feasible solution of $\Delta<0$. Then the solutions to the following equations

$$
\begin{aligned}
& \Sigma_{22}-\Omega_{2} \Delta^{-1} \Omega_{2}^{T}<0 \\
& \Sigma_{11}-\Omega_{1} \Delta^{-1} \Omega_{1}^{T}<0
\end{aligned}
$$

are given by

$$
\begin{aligned}
& {\left[\begin{array}{cc|c}
0 & \star \\
\hline\left[\begin{array}{cc}
I & 0 \\
0 & 0 \\
0 & D_{21}^{T} \\
0 & 0
\end{array}\right] & \Delta
\end{array}\right]\left[\begin{array}{c}
\hat{B}_{k 0}^{T} \\
\hat{B}_{k 1}^{T} \\
\hline *
\end{array}\right]=-\left[\begin{array}{c}
0 \\
\frac{C_{2}}{E^{T} \hat{D}_{k 11} C_{2}-\hat{H}_{1}} \\
-E^{T} B_{2}^{T} S+\hat{D}_{k 01} C_{2}-\hat{H}_{3} \\
B_{1}^{T} S \\
C_{1}+D_{12} \hat{D}_{k 11} C_{2}
\end{array}\right]} \\
& {\left[\begin{array}{c|c}
0 & \star \\
\hline\left[\begin{array}{cc}
0 & E^{T} \\
I & 0 \\
0 & 0 \\
0 & D_{12}
\end{array}\right] &
\end{array}\right]\left[\begin{array}{c}
\hat{C}_{k 0} \\
\hat{C}_{k 1} \\
\hline *
\end{array}\right]=-\left[\begin{array}{c}
0 \\
B_{2}^{T} \\
\hline L E^{T} B_{2}^{T}+\hat{D}_{k 10}^{T} B_{2}^{T}-\hat{H}_{2} \\
-E^{T} B_{2}^{T}-\hat{H}_{4} \\
B_{1}^{T}+D_{21}^{T} \hat{D}_{k 11}^{T} B_{2}^{T} \\
C_{1} R
\end{array}\right]}
\end{aligned}
$$

Proof: Using Schur complement, eqn. (33) is equivalent to

$$
\begin{aligned}
& \mathcal{F}\left(\Pi_{12}\right):=\left[\begin{array}{cc}
\Sigma_{22} & \Omega_{2} \\
\Omega_{2}^{T} & \Delta
\end{array}\right] \\
& =\left[\begin{array}{c|c}
A^{T} S+S A & \star \star \star \star \\
\hline E^{T} \hat{D}_{k 11} C_{2}-\hat{H}_{1} & \\
\hat{D}_{k 01} C_{2}-E^{T} B_{2}^{T} S-\hat{H}_{1 k} & \Delta \\
B_{1}^{T} S & \Delta \\
C_{1}+D_{12} \hat{D}_{k 11} C_{2} &
\end{array}\right]+\operatorname{He}\left\{\left[\begin{array}{cc}
0 & C_{2}^{T} \\
\hline I & 0 \\
0 & 0 \\
0 & D_{21}^{T} \\
0 & 0
\end{array}\right]\left[\begin{array}{c}
\hat{B}_{k 0}^{T} \\
\hat{B}_{k 1}^{T}
\end{array}\right]\left[\begin{array}{llll}
I \mid 0 & 0 & 0 & 0
\end{array}\right]\right\} \\
& :=\left[\begin{array}{cc}
A^{T} S+S A & \star \\
\bar{B}_{1} & \Delta
\end{array}\right]+\operatorname{He}\left\{\left[\begin{array}{c}
\bar{C}_{2}^{T} \\
\bar{D}_{21}^{T}
\end{array}\right] \Pi_{12}\left[\begin{array}{ll}
I & 0
\end{array}\right]\right\}<0,
\end{aligned}
$$

where $\Pi_{12}=\left[\begin{array}{ll}\hat{B}_{k 0} & \hat{B}_{k 1}\end{array}\right]^{T}$.

Defining $\phi\left(\Pi_{12} x, y\right)=\left[\begin{array}{ll}x^{T} & y^{T}\end{array}\right] \mathcal{F}\left(\Pi_{12}\right)\left[\begin{array}{l}x \\ y\end{array}\right]$, we have $\phi\left(\Pi_{12} x, y\right)<0$ hold for some $\Pi_{12}$ and any $(x, y) \neq 0$ if and only if

$$
\begin{aligned}
\phi(w, y)= & {\left[\begin{array}{c}
w \\
y
\end{array}\right]^{T}\left[\begin{array}{cc}
0 & \star \\
\bar{D}_{21}^{T} & \Delta
\end{array}\right]\left[\begin{array}{l}
w \\
y
\end{array}\right]+2\left[\begin{array}{l}
\bar{C}_{2} x \\
\bar{B}_{1} x
\end{array}\right]^{T}\left[\begin{array}{l}
w \\
y
\end{array}\right]+2 x^{T} S A x } \\
= & \left(\left[\begin{array}{cr}
0 & \star \\
\bar{D}_{21}^{T} & \Delta
\end{array}\right]\left[\begin{array}{c}
w \\
y
\end{array}\right]+\left[\begin{array}{l}
\bar{C}_{2} x \\
\bar{B}_{1} x
\end{array}\right]\right)^{T}\left[\begin{array}{cc}
0 & \star \\
\bar{D}_{21}^{T} & \Delta
\end{array}\right]^{-1}\left(\left[\begin{array}{cc}
0 & \star \\
\bar{D}_{21}^{T} & \Delta
\end{array}\right]\left[\begin{array}{c}
w \\
y
\end{array}\right]+\left[\begin{array}{l}
\bar{C}_{2} x \\
\bar{B}_{1} x
\end{array}\right]\right) \\
& -\left[\begin{array}{c}
\bar{C}_{2} x \\
\bar{B}_{1} x
\end{array}\right]^{T}\left[\begin{array}{cc}
0 & \star \\
\bar{D}_{21}^{T} & \Delta
\end{array}\right]^{-1}\left[\begin{array}{c}
\bar{C}_{2} x \\
\bar{B}_{1} x
\end{array}\right]+2 x^{T} S A x<0,
\end{aligned}
$$

where $w=\Pi_{12} x$. 
Since $D_{21}$ has full row rank and $\Delta$ is negative definite, the inverse matrix in eqn. (38) is welldefined. Moreover, the unique solution that minimizes the maximum value of function $\phi(w, y)$ is given by

$$
\left[\begin{array}{cc}
0 & \star \\
\bar{D}_{21}^{T} & \Delta
\end{array}\right]\left[\begin{array}{l}
w \\
y
\end{array}\right]=-\left[\begin{array}{l}
\bar{C}_{2} x \\
\bar{B}_{1} x
\end{array}\right]
$$

which is equivalent to

$$
\left[\begin{array}{cc}
0 & \star \\
\bar{D}_{21}^{T} & \Delta
\end{array}\right]\left[\begin{array}{c}
\Pi_{12} x \\
y
\end{array}\right]=-\left[\begin{array}{c}
\bar{C}_{2} x \\
\bar{B}_{1} x
\end{array}\right]
$$

Similar to the proof of Theorem 3.1 in [7], we have

$$
\phi\left(\Pi_{12} x, y\right)<0, \quad \forall(x, y) \neq 0
$$

when (39) holds. This verifies eqn. (35) as the solution of $\hat{B}_{k 0}, \hat{B}_{k 1}$.

Following the same procedure, one can obtain (36) as the solution of $\hat{C}_{k 0}, \hat{C}_{k 1}$. This completes the proof.

Q.E.D.

Therefore we obtain the following saturated controller construction procedure:

\section{Algorithm 1 (Controller reconstruction)}

1. Choosing $M, N$ and $U, V$ matrices such that $M N^{T}=I-R S, U V^{T}=I-L J$.

2. Solving $\hat{D}_{k 00}, \hat{D}_{k 01}, \hat{D}_{k 10}$ and $\hat{D}_{k 11}$ such that $\Delta<0$.

3. Determining $\hat{B}_{k 0}, \hat{B}_{k 1}$ and $\hat{C}_{k 0}, \hat{C}_{k 1}$ by solving eqns. (35) and (36).

4. Calculating $\hat{A}_{k}$ by zeroing the off-diagonal block $\Sigma_{21}-\Omega_{2} \Delta^{-1} \Omega_{1}^{T}$. This leads to

$$
\hat{A}_{k}=\Omega_{2} \Delta^{-1} \Omega_{1}^{T}-C_{2}^{T} \hat{D}_{k 11}^{T} B_{2}^{T}-A^{T}
$$

5. Computing the gain-scheduling controller original gains by

$$
\begin{aligned}
{\left[\begin{array}{ccc}
A_{k} & B_{k 0} & B_{k 1} \\
C_{k 0} & D_{k 00} & D_{k 01} \\
C_{k 1} & D_{k 10} & D_{k 11}
\end{array}\right] } & =\left[\begin{array}{ccc}
N & 0 & S B_{2} \\
0 & V & J E^{T} \\
0 & 0 & I
\end{array}\right]^{-1} \\
& \times\left[\begin{array}{ccc}
\hat{A}_{k}-S A R & \hat{B}_{k 0}+S B_{2} E L & \hat{B}_{k 1} \\
\hat{C}_{k 0} & \hat{D}_{k 00} & \hat{D}_{k 01} \\
\hat{C}_{k 1} & \hat{D}_{k 10} & \hat{D}_{k 11}
\end{array}\right]\left[\begin{array}{ccc}
M^{T} & 0 & 0 \\
0 & U^{T} & 0 \\
C_{2} R & 0 & I
\end{array}\right]^{-1}
\end{aligned}
$$

with

$$
\left[\begin{array}{cc}
H_{1} & H_{2} \\
H_{1 k} & H_{2 k}
\end{array}\right]=\left[\begin{array}{cc}
I & 0 \\
J & V
\end{array}\right]^{-1}\left[\begin{array}{cc}
\hat{H}_{1} & \hat{H}_{2} \\
\hat{H}_{1 k} & \hat{H}_{2 k}
\end{array}\right]\left[\begin{array}{cc}
I & R \\
0 & M^{T}
\end{array}\right]^{-1}
$$

For open-loop stable systems, the construction of output feedback saturation control follows the same procedure by setting $\hat{H}_{1}=\hat{H}_{2}=0$ and $\hat{H}_{1 k}=\hat{H}_{2 k}=0$. 
As mentioned before, in order to implement the output feedback gain-scheduling controller, we need to set $\hat{H}_{1}=\hat{H}_{1 k}=0$ in the control synthesis conditions (10)-(14) and the controller reconstruction scheme. However, as far as the performance is concerned, the use of restricted linear subspace could cause conservatism in the closed-loop performance, which means the performance of the output feedback gain-scheduled control system will be probably not as good as the full state feedback gain-scheduled control. When the open-loop system is stable, the linear subspace is not required and the proposed gain-scheduled control would achieve the optimal performance.

Remark 5 It is noted that the controller (6) in [4] is simply a special form of the proposed gainscheduling controller (5)-(6). When $H_{1}=H_{1 k}$ and $H_{2}=H_{2 k}$, we have

$$
\begin{aligned}
\operatorname{dz}(u) & =\Theta\left(u-H_{1} x-H_{2} x_{k}\right) \\
p_{k} & =\Theta\left(q_{k}-H_{1 k} x-H_{2 k} x_{k}\right) .
\end{aligned}
$$

Moreover, by letting $C_{k 1}=C_{k 0}, D_{k 10}=D_{k 00}, D_{k 11}=D_{k 01}$, we have $q_{k}=u$. As a result, the gain-scheduling controller becomes

$$
\begin{aligned}
{\left[\begin{array}{c}
\dot{x}_{k} \\
u
\end{array}\right] } & =\left[\begin{array}{ccc}
A_{k} & B_{k 0} & B_{k 1} \\
C_{k 0} & D_{k 00} & D_{k 01}
\end{array}\right]\left[\begin{array}{c}
x_{k} \\
p_{k} \\
y
\end{array}\right] \\
p_{k} & =\mathrm{dz}(u),
\end{aligned}
$$

which is exactly the controller form used in [4].

\section{$5 \quad$ Numerical Examples}

First, a modified inverted pendulum problem will be used to demonstrate the proposed gainscheduled saturation control approach (see Fig. 1). One motor is used to drive the base cart of the inverted pendulum. Different from a typical setup, another motor is installed on the moving cart to control the pendulum bar directly. This will provide a two-input controlled pendulum.

The linearized equations of motion for the inverted pendulum at its equilibrium $(0,0,0,0)$ are given by

$$
\left\{\begin{array}{l}
\dot{x}_{1}=x_{2} \\
\dot{x}_{2}=\frac{1}{c}\left[(M+m) m g l x_{1}-(M+m) f x_{2}+\mu m l x_{4}+(M+m) u_{1}-m l u_{2}+(M+m) d_{1}+m l d_{2}\right] \\
\dot{x}_{3}=x_{4} \\
\dot{x}_{4}=\frac{1}{c}\left[-m^{2} l^{2} g x_{1}+m l f x_{2}-\left(J+m l^{2}\right) \mu x_{4}-m l u_{1}+\left(J+m l^{2}\right) u_{2}+m l d_{1}+\left(J+m l^{2}\right) d_{2}\right]
\end{array}\right.
$$

where $c=(M+m)\left(J+m l^{2}\right)-m^{2} l^{2} . x_{1}, x_{2}, x_{3}$, and $x_{4}$ denote the angle of the bar from vertical axis, its angular velocity, the position of the cart along the linear track and its velocity. $d_{1}$ is the disturbance torque applied to the bar, and $d_{2}$ represents the disturbance force on the cart. $u_{1}, u_{2}$ represents the motor torque applied to the bar and the force exerted on the cart. It is assumed that control input $u_{1}$ is subject to saturation with its magnitude $\bar{u}_{1}=1 \mathrm{Nm}$. On the other hand, no saturation limit is imposed on the control input $u_{2}$. The angular position and car position will be measured outputs for control use. Therefore $y_{1}=x_{1}+n_{1}, y_{2}=x_{3}+n_{2}$, where $n_{1}, n_{2}$ are the measurement noises. The controlled outputs consist of performance variables $y_{1}, 0.1 y_{2}$ and penalty 


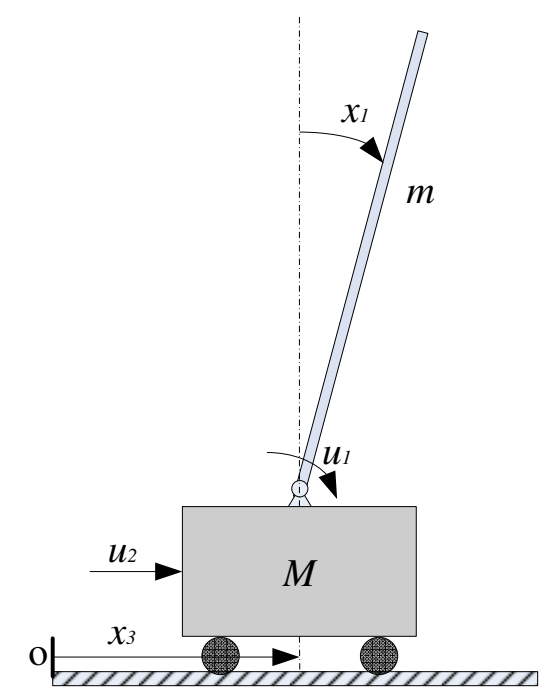

Figure 1: Schematic drawing of inverted pendulum.

on control inputs $u_{1}, u_{2}$. Our design objective is to stabilize the inverted pendulum and optimize the controlled performance with input saturation on $u_{1} . M$ and $m$ represent the mass of the cart and the bar respectively; $l$ is the half length of the bar; $J$ is bar's moment of inertia about its mass center; $\mu$ and $f$ are friction coefficients of the bar and the cart, respectively. The values of pendulum parameters are listed in Table 1.

Table 1: Parameters of the inverted pendulum.

\begin{tabular}{c|c}
\hline \hline parameter & value (units) \\
\hline$M$ & $1 \mathrm{~kg}$ \\
$m$ & $0.1 \mathrm{~kg}$ \\
$g$ & $9.8 \mathrm{~m} / \mathrm{sec}^{2}$ \\
$l$ & $0.4 \mathrm{~m}$ \\
$f$ & $0.01 \mathrm{~N} \cdot \mathrm{sec} / \mathrm{m}$ \\
$\mu$ & $0.01 \mathrm{~N} \cdot \mathrm{sec}$ \\
\hline \hline
\end{tabular}

As a result, the state-space model of the inverted pendulum will be

$$
\begin{aligned}
& {\left[\begin{array}{l}
\dot{x}_{1} \\
\dot{x}_{2} \\
\dot{x}_{3} \\
\dot{x}_{4}
\end{array}\right]=\left[\begin{array}{cccc}
0 & 1 & 0 & 0 \\
\frac{(M+m) m g l}{c} & -\frac{(M+m) f}{c} & 0 & \frac{\mu m l}{c} \\
0 & 0 & 0 & 1 \\
-\frac{m^{2} l^{2} g}{c} & \frac{m l f}{c} & 0 & -\frac{\left(J+m l^{2}\right) \mu}{c}
\end{array}\right]\left[\begin{array}{c}
x_{1} \\
x_{2} \\
x_{3} \\
x_{4}
\end{array}\right]+\left[\begin{array}{cccc}
0 & 0 & 0 & 0 \\
\frac{M+m}{c} & \frac{m l}{c} & \frac{M+m}{c} & -\frac{m l}{c} \\
0 & 0 & 0 & 0 \\
\frac{m l}{c} & \frac{J+m l^{2}}{c} & -\frac{m l}{c} & \frac{J+m l^{2}}{c}
\end{array}\right]\left[\begin{array}{c}
d_{1} \\
d_{2} \\
\operatorname{sat}\left(u_{1}\right) \\
u_{2}
\end{array}\right]} \\
& {\left[\begin{array}{l}
e_{1} \\
e_{2} \\
e_{3} \\
e_{4} \\
y_{1} \\
y_{2}
\end{array}\right]=\left[\begin{array}{llll}
1 & 0 & 0 & 0 \\
0 & 0 & 0.1 & 0 \\
0 & 0 & 0 & 0 \\
0 & 0 & 0 & 0 \\
1 & 0 & 0 & 0 \\
0 & 0 & 1 & 0
\end{array}\right]\left[\begin{array}{l}
x_{1} \\
x_{2} \\
x_{3} \\
x_{4}
\end{array}\right]+\left[\begin{array}{cccc}
1 & 0 & 0 & 0 \\
0 & 0.1 & 0 & 0 \\
0 & 0 & 1 & 0 \\
0 & 0 & 0 & 1 \\
1 & 0 & 0 & 0 \\
0 & 1 & 0 & 0
\end{array}\right]\left[\begin{array}{c}
n_{1} \\
n_{2} \\
\operatorname{sat}\left(u_{1}\right) \\
u_{2}
\end{array}\right] .}
\end{aligned}
$$


It is easy to verify that the open-loop system is unstable because it contains one positive pole at 4.38 .

By solving the synthesis condition (10)-(14), we determine a suboptimal performance level of $\gamma=$ 6.0518 for $s=0.1$. The following controller matrices are also obtained using controller construction algorithm with a condition number of 582.21 .

$$
\begin{aligned}
\Pi & =\left[\begin{array}{rrrr|r|rr}
-14.9347 & 1.1630 & -0.5608 & -0.1690 & -0.0003 & 15.2307 & 0.5573 \\
-9.6699 & -6.9713 & -0.1163 & -1.3548 & -0.0039 & -9.9841 & -0.0472 \\
-0.5279 & 0.0121 & -1.9416 & 0.9862 & -0.0000 & 0.5680 & 1.9408 \\
-3.2751 & 0.3680 & -1.9461 & -0.4613 & -0.0000 & 4.4724 & 1.8850 \\
\hline-0.9266 & 0.6518 & -0.1158 & 0.0914 & 1.0000 & -2.1383 & -0.1220 \\
\hline 1.2166 & -0.1762 & 0.0979 & -0.1192 & 1.0000 & -2.1383 & -0.1220 \\
1.2872 & -0.0539 & 0.1527 & -0.8287 & -0.0000 & -1.3417 & -0.2936
\end{array}\right] \\
H_{2} & =\left[\begin{array}{llll}
-0.6726 & -0.0976 & -0.0248 & -0.1799
\end{array}\right] \\
H_{2 k} & =\left[\begin{array}{llll}
-2.8158 & 0.7304 & -0.2386 & 0.0307
\end{array}\right]
\end{aligned}
$$

with $H_{1}=H_{1 k}=0$.

In our simulation, the disturbance $d_{1}$ is chosen as a pulse force starting at $5 \mathrm{sec}$ and ending at $6 \mathrm{sec}$, and other disturbances are all set to 0 . The response of the closed-loop system for $s=0.1$ case is shown in Fig. 2 with the disturbance magnitude of $0.42 \mathrm{Nm}$.

As suggested by the main theorem, one can obtain controllers that tolerate a larger class of disturbances with higher energy level by increasing the value of $s$. Nevertheless, the performance of the closed-loop system will degrade as seen from Table 2. Note that the condition number of the resulting controllers is not necessarily increasing monotonically.

Table 2: Relation between disturbance level $s$ and performance $\gamma$.

\begin{tabular}{c|c|c}
\hline \hline disturbance $s$ & performance $\gamma$ & condition number \\
\hline 0.001 & 4.6055 & $7.9136 \mathrm{e}+003$ \\
0.01 & 4.6111 & $4.4872 \mathrm{e}+003$ \\
0.05 & 4.7569 & $3.5218 \mathrm{e}+003$ \\
0.1 & 6.0518 & 582.2141 \\
0.2 & 47.7136 & $2.3676 \mathrm{e}+003$ \\
0.4 & 63.3424 & $8.2740 \mathrm{e}+005$ \\
0.6 & 67.3140 & $2.2187 \mathrm{e}+006$ \\
0.8 & 69.3377 & $1.6787 \mathrm{e}+006$ \\
1.0 & 70.5629 & $8.3089 \mathrm{e}+005$ \\
\hline \hline
\end{tabular}

When the value of $s$ increases to 1 , the relaxed performance level grows to $\gamma=70.5629$. The 


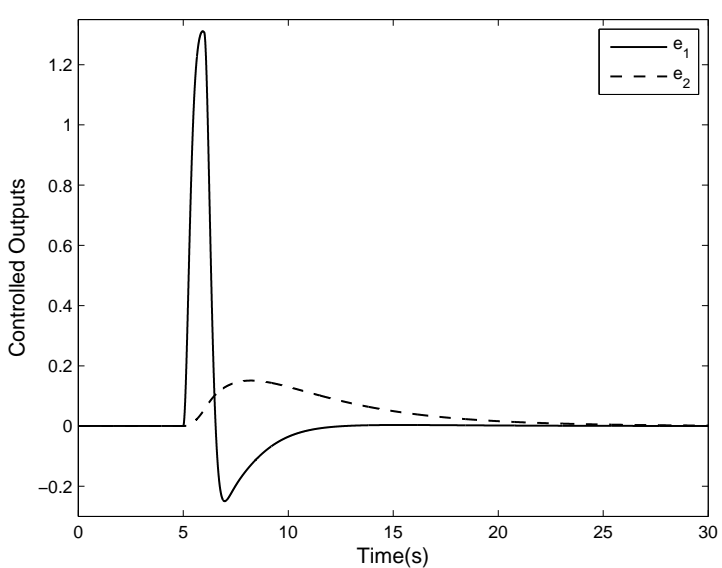

(a) output $e$

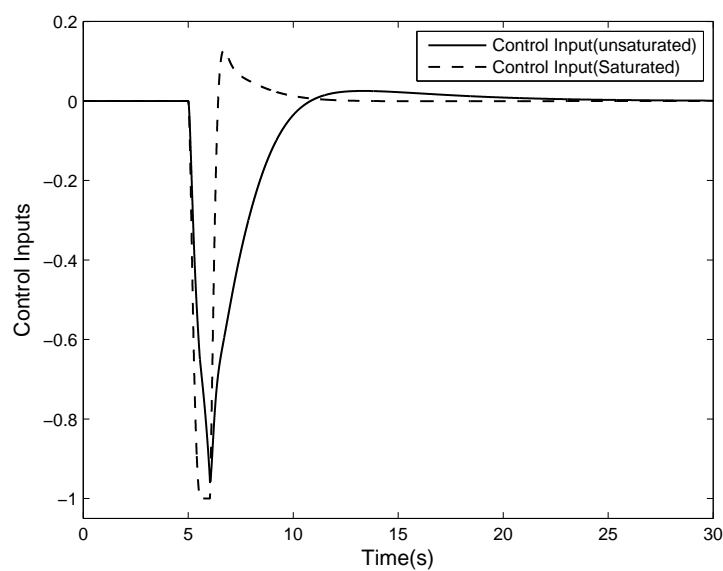

(b) control $u$

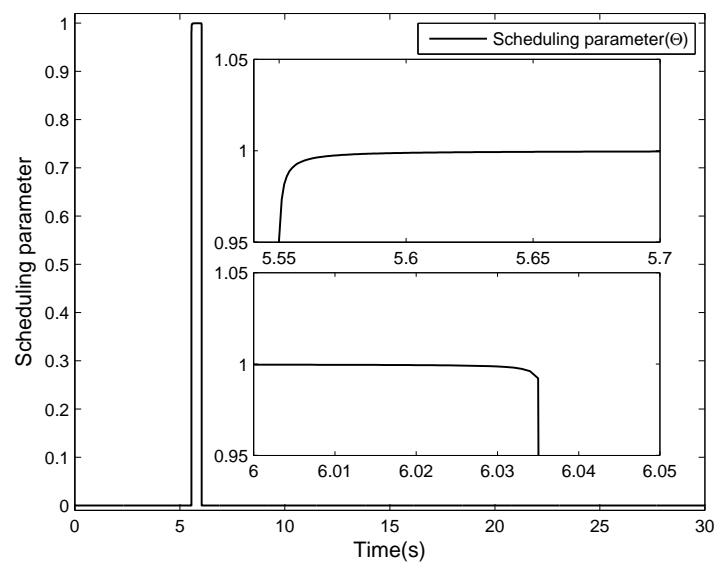

(c) parameter $\theta$

Figure 2: Response of the inverted pendulum: $s=0.1$ case.

obtained controller matrices have a condition number of $8.3089 e+005$.

$$
\begin{aligned}
\Pi & =10^{4} \times\left[\begin{array}{rrrr|r|rr}
0.0611 & -0.1846 & -0.0041 & -0.0036 & -0.0000 & 0.0241 & 0.0011 \\
0.4684 & -1.3806 & -0.0302 & -0.0269 & -0.0000 & 0.1657 & 0.0076 \\
0.0019 & -0.0058 & -0.0003 & -0.0000 & -0.0000 & 0.0007 & 0.0002 \\
0.0112 & -0.0337 & -0.0009 & -0.0007 & -0.0000 & 0.0044 & 0.0004 \\
\hline-0.0003 & 0.0000 & -0.0000 & 0.0000 & 0.0001 & -0.0003 & -0.0000 \\
\hline 0.0002 & -0.0000 & 0.0000 & -0.0000 & 0.0001 & -0.0003 & -0.0000 \\
0.0002 & -0.0000 & -0.0000 & -0.0000 & -0.0000 & -0.0004 & -0.0000
\end{array}\right] \\
H_{2} & =\left[\begin{array}{lllll}
0.0012 & 0.0001 & -0.0023 & -0.0101
\end{array}\right] \\
H_{2 k} & =\left[\begin{array}{lllll}
-4.2288 & 0.5660 & -0.2011 & 0.0328
\end{array}\right]
\end{aligned}
$$

with $H_{1}=H_{1 k}=0$.

This controller can tolerate much larger disturbance than the one for $s=0.1$. Using the controller designed for $s=1$, the closed-loop system remains stable for the disturbance up to $3.06504 \mathrm{Nm}$. 
However, for $s=0.1$, the closed-loop system becomes unstable when the magnitude of $d_{1}$ increases to $0.47678 \mathrm{Nm}$. The corresponding response of the closed-loop system for the case of $s=1$ is shown in Fig. 3 with the disturbance magnitude of $1.5 \mathrm{Nm}$.

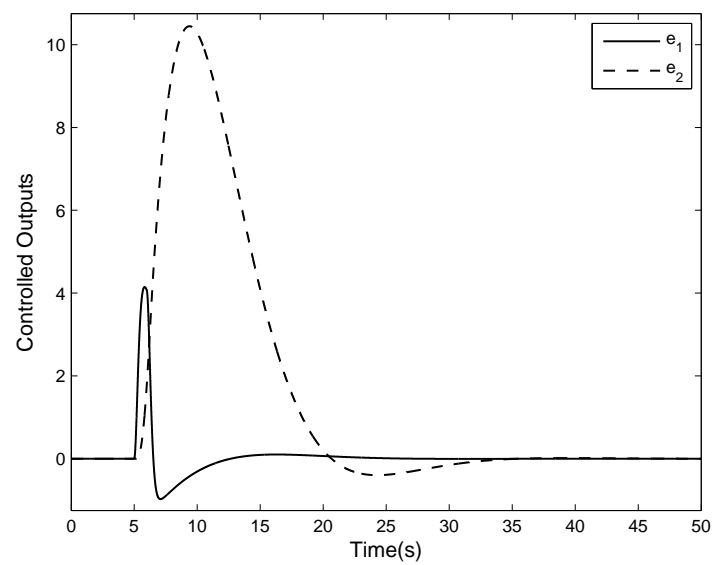

(a) output $e$

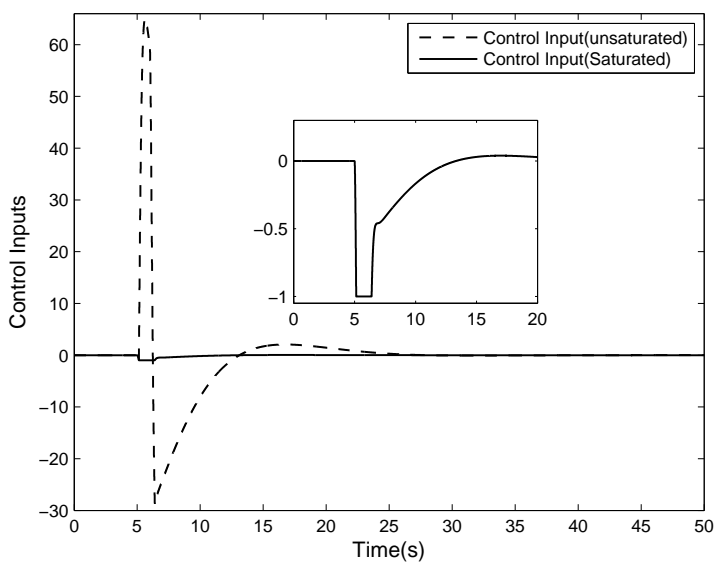

(b) control $u$

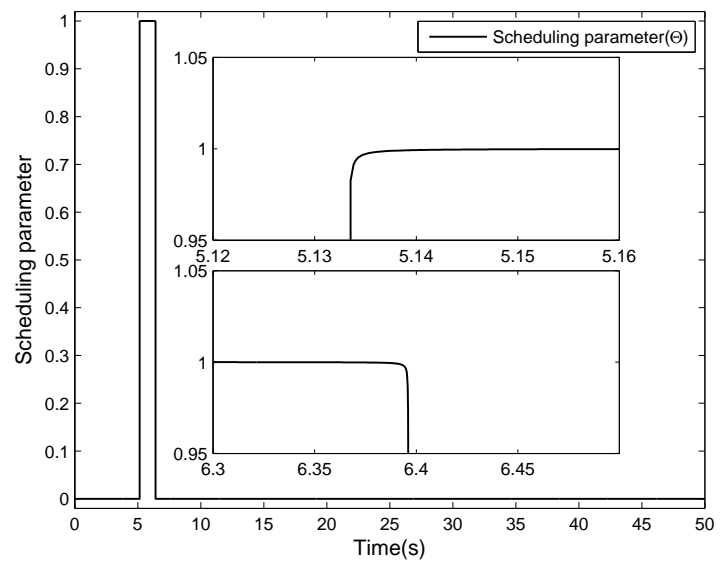

(c) parameter $\theta$

Figure 3: Response of the inverted pendulum: $s=1$ case.

As suggested in the introduction, the results developed in this article can also help to analyze which input channel saturation will have a stronger influence on the performance of the closedloop system. To this end, we will first assume $u_{1}$ subject to saturation with $u_{2}$ free of constraints and calculate the performance $\gamma$ as listed in Table 3. Then $u_{2}$ is assumed to be under actuator saturation and $u_{1}$ is not subject to any saturation constraint, as shown in Table 4 . It is observed that with the growth of the saturation level, the performance for a fixed disturbance level $s$ will improve in both cases and almost reach the same value 4.6054 at the end. This means if all the input channels are not subject to saturation, the ultimate performance $\gamma$ will be 4.6054 . However, when $u_{1}$ is subject to saturation, the performance will increase to about 75.5610. When $u_{2}$ is subject to saturation, the situation will be much worse. In fact, the performance for $s=0.0001$ will degrade to 122.7227 with $\bar{u}_{2}=0.0001$ and no stabilizing controller can even be found with a smaller saturation level. This phenomenon is more obvious with increased disturbance level $s$. 
Therefore, one can conclude that the limitation of force exerted on the cart, i.e., $\bar{u}_{2}$, will have a stronger influence on the closed-loop performance. Hence to get a better performance, we should better select an actuator with a larger saturation level for the input channel $u_{2}$.

Table 3: Relation between saturation level $\bar{u}_{1}$ and performance $\gamma$.

\begin{tabular}{l|c|c|c}
\hline \hline saturation level $\bar{u}_{1}$ & performance $\gamma(\mathrm{s}=0.0001)$ & performance $\gamma(\mathrm{s}=0.1)$ & performance $\gamma(\mathrm{s}=1)$ \\
\hline 0.00001 & 75.0535 & 75.5605 & 75.5610 \\
0.0001 & 70.5629 & 75.5559 & 75.5605 \\
0.001 & 6.0519 & 75.5102 & 75.5560 \\
0.01 & 4.6111 & 75.0536 & 75.5102 \\
0.1 & 4.6055 & 70.5629 & 75.0535 \\
1 & 4.6054 & 6.0518 & 70.5629 \\
10 & 4.6054 & 4.6111 & 6.0519 \\
100 & 4.6054 & 4.6055 & 4.6111 \\
1000 & 4.6054 & 4.6054 & 4.6055 \\
\hline \hline
\end{tabular}

Table 4: Relation between saturation level $\bar{u}_{2}$ and performance $\gamma$.

\begin{tabular}{l|c|c|c}
\hline \hline saturation level $\bar{u}_{2}$ & performance $\gamma(\mathrm{s}=0.0001)$ & performance $\gamma(\mathrm{s}=0.1)$ & performance $\gamma(\mathrm{s}=1)$ \\
\hline 0.00001 & - & - & - \\
0.0001 & 122.7227 & - & - \\
0.001 & 4.6398 & - & - \\
0.01 & 4.6066 & - & - \\
0.1 & 4.6054 & 122.7227 & 122.7227 \\
1 & 4.6054 & 4.6398 & 4.6398 \\
10 & 4.6054 & 4.6066 & 4.6066 \\
100 & 4.6054 & 4.6054 & 4.6054 \\
1000 & 4.6054 & 4.6054 & $\bar{y}^{2}$ \\
\hline \hline
\end{tabular}

As the second example, we consider a stable plant of the linearized longitudinal dynamics of the F8 aircraft in [12]. Its state equations are given by

$$
\begin{aligned}
& {\left[\begin{array}{c}
\dot{q} \\
\dot{v} \\
\dot{\alpha} \\
\dot{\theta}
\end{array}\right]=\left[\begin{array}{cccc}
-0.8 & -0.0006 & -12 & 0 \\
0 & -0.014 & -16.64 & -32.2 \\
1 & -0.0001 & -1.5 & 0 \\
1 & 0 & 0 & 0
\end{array}\right]\left[\begin{array}{l}
q \\
v \\
\alpha \\
\theta
\end{array}\right]+\left[\begin{array}{cc}
-19 & -3 \\
-0.66 & -0.5 \\
-0.16 & -0.5 \\
0 & 0
\end{array}\right]\left[\begin{array}{l}
\operatorname{sat}\left(\delta_{e}\right) \\
\operatorname{sat}\left(\delta_{f}\right)
\end{array}\right]} \\
& {\left[\begin{array}{l}
\theta \\
\gamma
\end{array}\right]=\left[\begin{array}{cccc}
0 & 0 & 0 & 1 \\
0 & 0 & -1 & 1
\end{array}\right]\left[\begin{array}{l}
q \\
v \\
\alpha \\
\theta
\end{array}\right]}
\end{aligned}
$$

where $q, v, \alpha$ and $\theta$ denote the pitch rate $(\mathrm{rad} / \mathrm{sec})$, the forward velocity $(\mathrm{ft} / \mathrm{sec})$, the angle of attack ( $\mathrm{rad})$ and the pitch angle $(\mathrm{rad}) ; \gamma$ represents the flight path angle $(\mathrm{rad}) ; \delta_{e}$ and $\delta_{f}$ represent the elevator angle (deg) and the flaperon angle (deg). Both of them are subject to the actuator saturation with the limit $\bar{\delta}_{e}=\bar{\delta}_{f}=25^{\circ}$. This model is used as a benchmark to compare our synthesis approach with the output feedback control method in [4]. We intend to design a controller which can attenuate the disturbances as much as possible. For this purpose, four fictitious disturbances 
are introduced into the system. The augmented open-loop system is given by

$$
\begin{aligned}
& {\left[\begin{array}{c}
\dot{q} \\
\dot{v} \\
\dot{\alpha} \\
\dot{\theta}
\end{array}\right]=\left[\begin{array}{cccc}
-0.8 & -0.0006 & -12 & 0 \\
0 & -0.014 & -16.64 & -32.2 \\
1 & -0.0001 & -1.5 & 0 \\
1 & 0 & 0 & 0
\end{array}\right]\left[\begin{array}{c}
q \\
v \\
\alpha \\
\theta
\end{array}\right]+\left[\begin{array}{cccc}
1 & 0 & -19 & -3 \\
0 & 1 & -0.66 & -0.5 \\
0 & 0 & -0.16 & -0.5 \\
0 & 0 & 0 & 0
\end{array}\right]\left[\begin{array}{c}
d_{1} \\
d_{2} \\
\operatorname{sat}\left(\delta_{e}\right) \\
\operatorname{sat}\left(\delta_{f}\right)
\end{array}\right]} \\
& {\left[\begin{array}{c}
e_{1} \\
e_{2} \\
e_{3} \\
e_{4} \\
\theta \\
\gamma
\end{array}\right]=\left[\begin{array}{cccc}
0 & 0 & 0 & 0.1 \\
0 & 0 & -0.1 & 0.1 \\
0 & 0 & 0 & 0 \\
0 & 0 & 0 & 0 \\
0 & 0 & 0 & 1 \\
0 & 0 & -1 & 1
\end{array}\right]\left[\begin{array}{l}
q \\
v \\
\alpha \\
\theta
\end{array}\right]+\left[\begin{array}{cccc}
0.01 & 0 & 0 & 0 \\
0 & 0.01 & 0 & 0 \\
0 & 0 & 0.001 & 0 \\
0 & 0 & 0 & 0.001 \\
0.1 & 0 & 0 & 0 \\
0 & 0.1 & 0 & 0
\end{array}\right]\left[\begin{array}{c}
n_{1} \\
n_{2} \\
\operatorname{sat}\left(\delta_{e}\right) \\
\operatorname{sat}\left(\delta_{f}\right)
\end{array}\right] .}
\end{aligned}
$$

Using the proposed approach, we obtain the optimal performance level $\gamma=1.5205$. As a comparison, the optimal performance level obtained from Dai's method is 1.52084. Thus the results from two methods are essentially the same. With some constraints on the magnitude of controller gains, we obtain a suboptimal performance level of $\gamma=1.5208$ with the following controller matrices

$\Pi=\left[\begin{array}{rrrr|rr|rr}-191.7010 & 2.7240 & -66.6127 & -146.0940 & 0.0230 & 0.3914 & -117.8261 & -24.6985 \\ -12.3362 & 0.3769 & -101.4133 & 50.1876 & 0.0029 & 0.0512 & -29.2991 & 20.0321 \\ -9.7641 & 0.3748 & -58.0123 & 15.6398 & 0.0028 & 0.0488 & 11.4751 & 51.1400 \\ 0.8352 & -0.0004 & 1.6225 & -4.4945 & 0.0000 & 0.0000 & 2.7412 & 0.3102 \\ \hline 7.0117 & -0.0263 & -15.9224 & 13.9512 & 0.9997 & -0.0055 & 9.8585 & 18.3012 \\ 19.1010 & -0.7419 & 119.3951 & -40.9429 & -0.0055 & 0.9042 & -22.3279 & -108.3123 \\ \hline 7.0117 & -0.0263 & -15.9224 & 13.9512 & 0.9997 & -0.0055 & 9.8585 & 18.3012 \\ 19.1010 & -0.7419 & 119.3951 & -40.9429 & -0.0055 & 0.9042 & -22.3279 & -108.3123\end{array}\right]$.

For this controller, the poles of the closed loop system are at $-194.80,-104.77,-2.16+3.81 i,-2.16-$ $3.81 i,-1.71,-1.13,-0.63-0.01$. The corresponding response of the closed-loop system is shown in Figs. 5, in which the magnitude of the disturbance is 100 starting at $5 \mathrm{sec}$ and ending at $6 \mathrm{sec}$.

\section{Conclusions}

In this article, a new gain-scheduling control approach is proposed to design output feedback controllers for linear plants with partial input channels saturated. The control synthesis condition is formulated as a convex optimization in terms of LMIs. In the control design process, the Lyapunov matrices are first solved by LMI optimizations, controller gains are then obtained by an explicit construction algorithm. For unstable plants, if the solution to the LMIs does exist, a gain-scheduling output feedback controller can be constructed that guarantees regional stability of the closed-loop system and achieves prescribed disturbance/error attenuation performance. As for stable plants, a globally stabilizing output feedback control could be obtained. The gain-scheduling control design provides an alternative approach to the existing actuator saturation control methods. More importantly, the proposed approach can be easily extended to linear parameter-varying systems with saturated inputs and would broaden the applicability of the proposed method. 


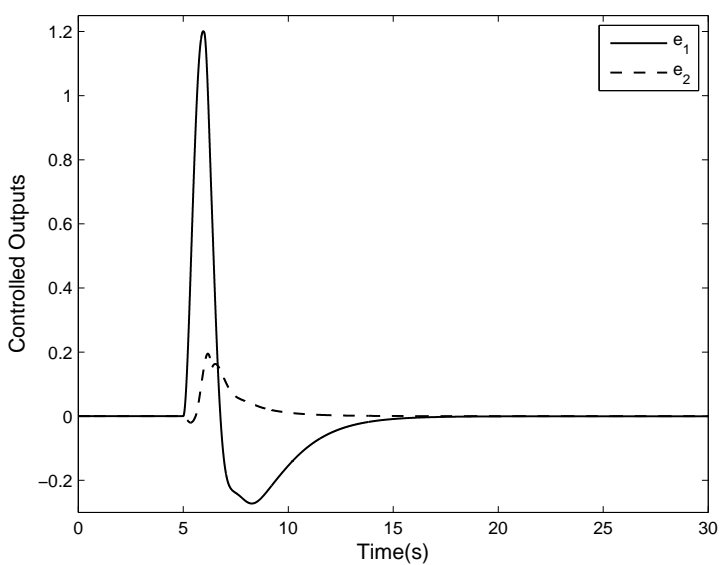

(a) output $e$

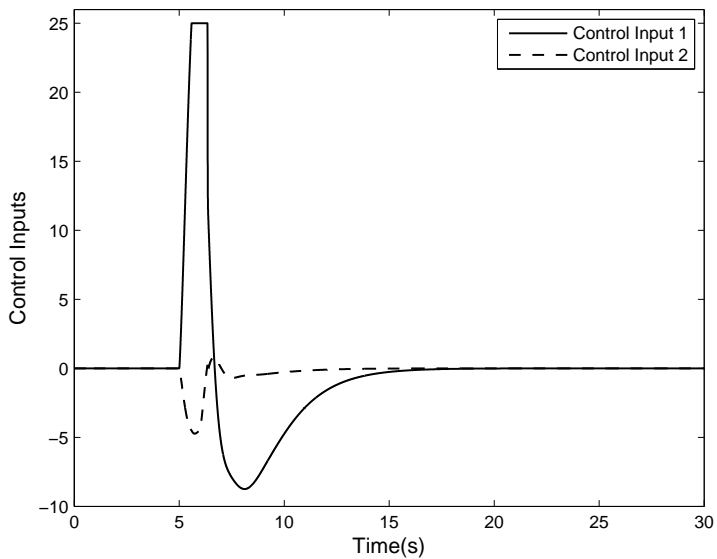

(b) control $u$

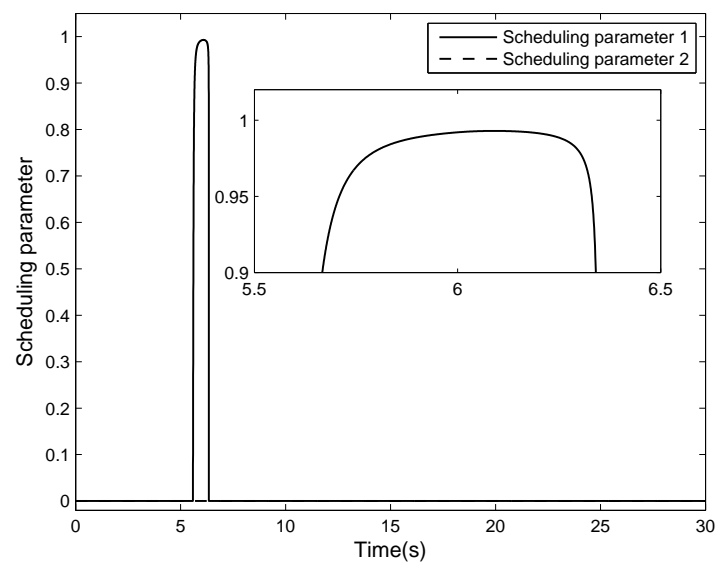

(c) parameter $\theta$

Figure 4: Response of the F-8 model.

\section{Acknowledgements}

Xiaojun Ban's work is supported by the National Natural Science Foundation of China (NSFC) under Grant No. 61304006 and 61273095. Fen Wu's work is supported in part by the NSF Grant CMMI-0800044.

\section{References}

[1] P. Apkarian and P. Gahinet, "A convex characterization of gain-scheduled $\mathcal{H}_{\infty}$ controllers," IEEE Trans. Automat. Contr., 40:853-864, 1995. Also see Erratum in IEEE Trans. Automat. Contr., 40:1681.

[2] D.S. Bernstein and A.N. Michel, "A chronological bibliography on saturating actuators," Int. J. Robust Non. Contr., 5:375-380, 1995. 
[3] Y. Chitour, "On the $L_{p}$ stabilization of the double integrator subject to input saturation," ESAIM: Control, Optimisation and Calculus of Variations, 6:291-331, 2001.

[4] D. Dai, T. Hu, A.R. Teel, and L. Zaccarian, "Output feedback design for saturated linear plants using deadzone loops," Automatica, 45:2917-2924, 2009.

[5] H. Fang, Z. Lin, and T. Hu, "Analysis of linear systems in the presence of actuator saturation and $\mathcal{L}_{2}$-disturbances," Automatica, 40(7):1229-1238, 2004.

[6] P. Gahinet and P. Apkarian, "A linear matrix inequality approach to $\mathcal{H}_{\infty}$ control," Int. J. Robust Non. Contr., 4:421-448, 1994.

[7] P. Gahinet, "Explicit Controller Formulas for LMI-based $\mathcal{H}_{\infty}$ synthesis," Automatica, 32(7):1007-1014, 1996.

[8] H. Hindi and S. Boyd, "Analysis of linear systems with saturation using convex optimization," in Proc. 37th IEEE Conf. Dec. Contr., Tempa, FL, 1998, pp. 903-908.

[9] T. Hu and Z. Lin, Control Systems with Actuator Saturation: Analysis and Design, Birkhauser: Boston, MA, 2001.

[10] T. Hu and Z. Lin, "Output regulation of linear systems with bounded continuous feedback," IEEE Trans. Automat. Contr. 49(11):1941-1953, 2004.

[11] T. Hu, A.R. Teel, and L. Zaccarian, "Stability and performance for saturated systems via quadratic and non-quadratic Lyapunov functions," IEEE Trans. Automat. Contr., 51(11):1770-1786, 2006.

[12] P. Kapasouris, M. Athans, and G. Stein, "Design of feedback control systems for stable plants with saturating actuators," in Proc. 27th IEEE Conf. Dec. Contr., 1988, pp. 469-479.

[13] H. K. Khalil, Nonlinear Systems, Prentice hall: New Jersey, USA, 2002.

[14] Z. Lin, Low Gain Feedback, Springer: London, UK, 1998.

[15] Z. Lin, A. Saberi, and A.R. Teel, "Almost disturbance decoupling with internal stability for linear systems subject to input saturation-State feedback case," Automatica, 32(4):619-624, 1996.

[16] W. Liu, Y. Chitour, and E. Sontag, "On finite gain stabilizability of linear systems subject to input saturation," SIAM J. Contr. Optimization, 34(4):1190-1219, 1996.

[17] T. Nguyen and F. Jabbari, "Disturbance attenuation for systems with input saturation: An LMI approach," IEEE Trans. Auto. Contr., 44(4):852-857, 1999.

[18] A. Packard, "Gain scheduling via linear fractional transformations," Syst. Contr. Letts., 22:7992, 1994.

[19] C. Paim, S. Tarbouriech, J.M. Gomes da Silva Jr., and E.B. Castelan, "Control design for linear systems with saturating actuators and $\ell_{2}$-bounded disturbances," in Proc. 41st IEEE Conf. Dec. Contr., Las Vegas, NV, 2002; pp. 4148-4153.

[20] A. Saberi, Z. Lin, and A.R. Teel, "Control of linear systems with saturating actuators," IEEE Trans. Automat. Contr., 41(3):368-378, 1996. 
[21] G. Scorletti, J.P. Folcher, and L. El Ghaoui, "Output feedback control with input saturations: LMI design approaches," Euro. J. Contr., 7(6), 2002.

[22] H.J. Sussmann, E.D. Sontag, and Y. Yang, "A general result on the stabilization of linear systems using bounded controls," IEEE Trans. Automat. Contr., 39(12):2411-2425, 1994.

[23] S. Tarbouriech and G. Garcia (eds), Control of Uncertain Systems with Bounded Inputs, Springer: London, UK, 1997.

[24] F. Wu, "Convexified Robust Control Approach and Its Application to Actuator Saturation Control," in Proc. IEEE Conf. Contr. Appl., 2004, pp. 1189-1194.

[25] F. Wu, Z. Lin, and Q. Zheng, "Output feedback stabilization of linear systems with actuator saturation," IEEE Trans. Automat. Contr., 52(1):122-128, 2007.

[26] F. Wu, Q. Zheng, and Z. Lin, "Disturbance attenuation by output feedback for linear systems subject to actuator saturation," Int. J. Robust Non. Contr., 19:168-184, 2009.

[27] F. Wu, "New results on output feedback disturbance attenuation of saturated linear systems," in Proc. 30st Chinese Control Conf., Yantai, China, 2011, pp. 287-292. 